\title{
Décadrages Décadrages
}

cinéma, à travers champs Cinéma, à travers champs

$16-17$ | 2010

Les abîmes de l'adaptation

\section{Entre génie et Ophélie : images de Virginia Woolf dans The Hours}

Rachel Noël

\section{(2) OpenEdition}

Journals

Édition électronique

URL : https://journals.openedition.org/decadrages/238

DOI : $10.4000 /$ decadrages. 238

ISSN : 2297-5977

Éditeur

Association Décadrages

Édition imprimée

Date de publication : 1 février 2010

Pagination : 71-94

ISBN : 978-2-9700668-1-1

ISSN : 2235-7823

\section{Référence électronique}

Rachel Noël, «Entre génie et Ophélie : images de Virginia Woolf dans The Hours », Décadrages [En ligne], 16-17 | 2010, mis en ligne le 10 février 2011, consulté le 03 avril 2022. URL : http://

journals.openedition.org/decadrages/238; DOI : https://doi.org/10.4000/decadrages.238 


\section{Entre génie et Ophélie: images romantiques}

\author{
de Virginia Woolf dans The Hours
}

\author{
par Rachel Noël
}

Un sombre courant fluvial, ininterrompu, est d'abord perçu en travelling arrière. S'y surimpressionne ensuite un titre, "Sussex, England, 1941 ». Enfin, la caméra s’élève pour dévoiler une rivière agitée, bordée d'arbres. C'est sur ces images que s'ouvre The Hours (Stephen Daldry, Etats-Unis, 2002), en un prologue de pré-générique dédié aux derniers instants de la vie de Virginia Woolf $\mathbf{1}$, que l'on suivra jusqu'au moment de son suicide par noyade. Cette séquence d'ouverture du biopic recourt à ce qui constitue l'un des principes clé du film: l'entrelacement des temporalités. Ainsi le dernier trajet de l'héroïne, traversé de gestes brefs et décidés, est-il monté en alternance avec deux autres actions : celle, située antérieurement, de la rédaction d'une lettre d'adieu de l'écrivaine à son époux et celle de la découverte, un peu plus tard, de cette même missive par le mari rentrant au foyer. Ce segment inaugural s'achève sur la disparition de Woolf dans les flots: le corps de l'écrivaine coule à pic avant d'être irrésistiblement emporté par le courant, malgré le poids des pierres qui lestent ses poches, en une navigation inconsciente (fig. 1-8). Trois plans sous-marins, graduellement fragmentés - vue indistincte, glissant progressivement du haut vers la partie inférieure du corps, puis deux détails successifs des mains aux paumes ouvertes et des pieds qui se déchaussent - insistent alors sur le détachement graduel de l'être humain vis-à-vis du monde matériel: le déplacement régulier de cette enveloppe charnelle, de ce corps libéré, comme rendu à l'espace naturel, se calque en quelque sorte sur le mouvement "cosmique» de l'eau (fig. 9-11).

Ce prologue expose, d'entrée de jeu, certains éléments de structure importants et met aussitôt l'accent sur l'acte d'écriture ainsi que sur sa réception. Cette étude se concentrera sur la représentation cinématographique de Virginia Woolf, une figuration qui me paraît ambivalente puisque, bien que d'emblée rattachée à l'icône victorienne d'Ophélie, l'écrivaine anglaise est pourtant presque simultanément ancrée dans le
1 Hormis cette mention introductive, j'utiliserai les dénominations "Virginia" ou "Woolf" pour qualifier le personnage du film, réservant les termes "Virginia Woolf" pour faire référence à la romancière et essayiste anglaise (18821941), figure littéraire marquante du XXe siècle. 

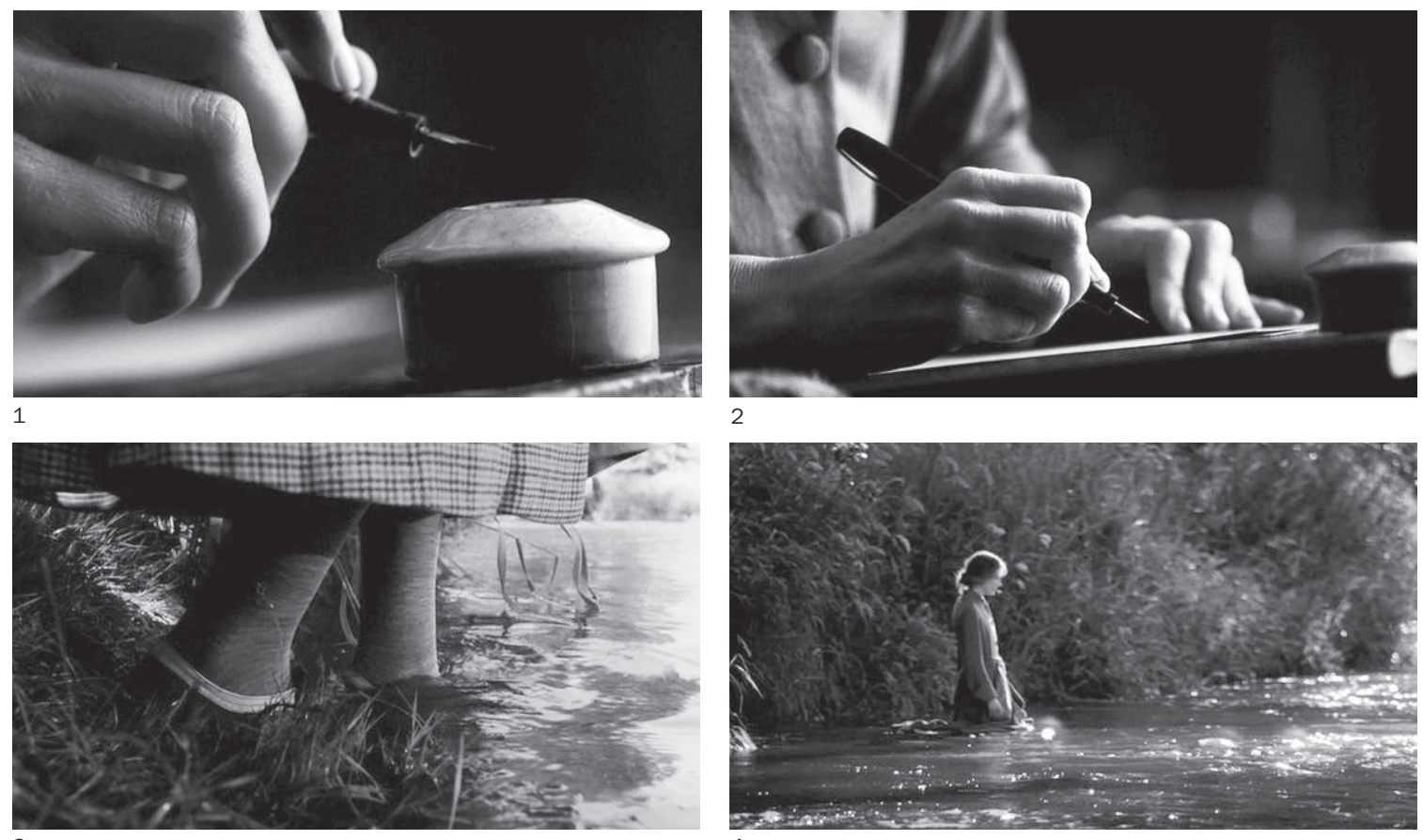

2

3
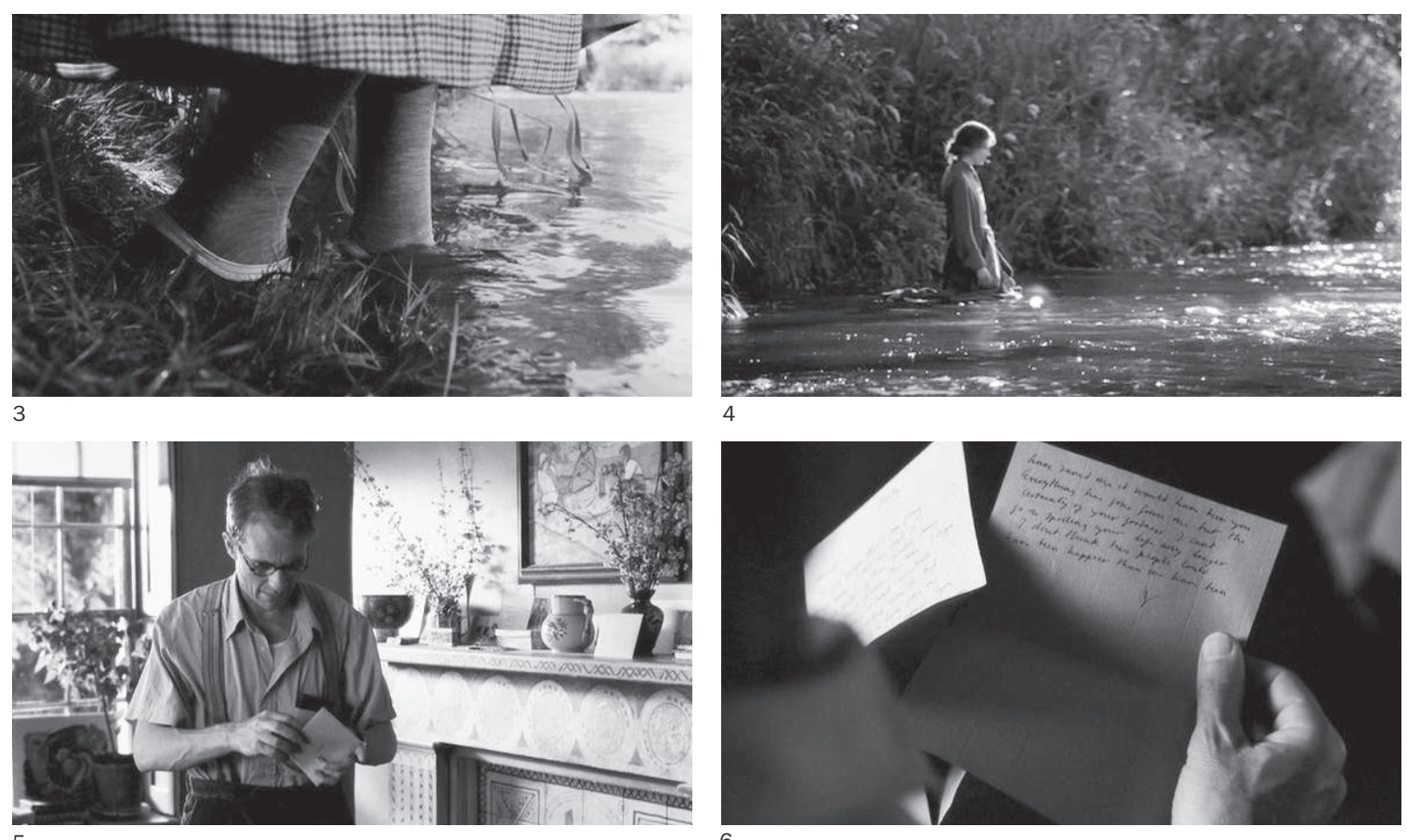
4
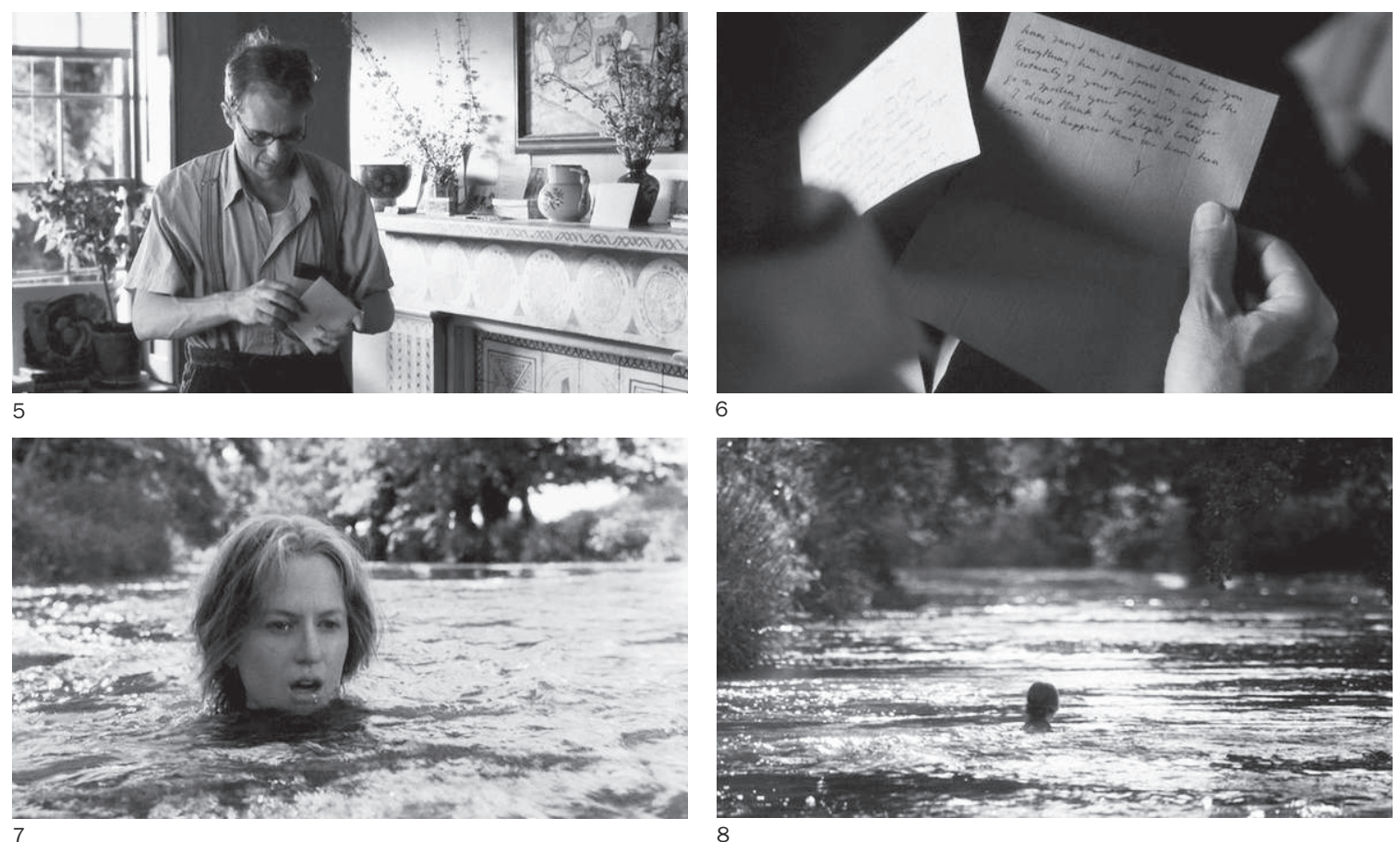

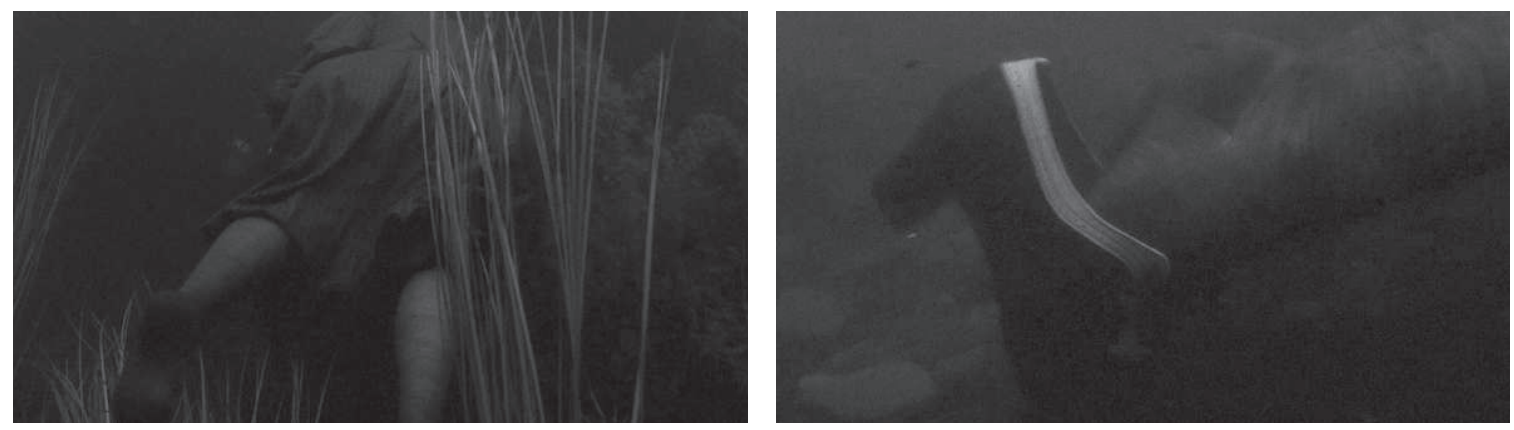

9

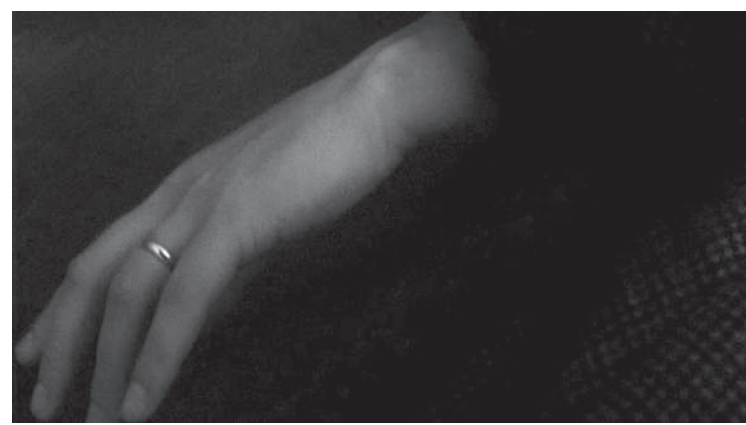

11

quotidien d'une journée durant laquelle elle entame l'écriture d'un nouveau roman. Le processus créatif fait alors l'objet de mises en relation élaborées entre la figure de l'artiste "réelle», les personnages tirés de son univers fictionnel, et certains motifs issus de son œuvre - plus précisément ceux qui associent mort et élément aquatique.

Si le montage de cet incipit mêle trois étapes rapprochées d'une même journée de 1941, cette micro-alternance annonce en fait un autre croisement chronologique, essentiel au propos du film et qui apparaît dès le générique: celui qui unit le destin de trois femmes d'époques différentes. Adaptation d'un roman postmoderne 2 de Michael Cunningham qui porte le même titre, The Hours entretient une relation intertextuelle complexe et foisonnante autant avec l'œuvre de Virginia Woolf qu'avec la propre biographie de l'auteure anglaise. Il fait en effet directement référence à l'un de ses textes les plus célèbres : Mrs Dalloway ${ }^{3}$. Ce roman narre une journée de l'existence de Clarissa Dalloway, dame de la haute bourgeoisie du début du XX $\mathrm{XX}^{\mathrm{e}}$ siècle qui s'affaire à la préparation d'une fête prévue le soir même et qui rencontre, durant ses emplettes, des individus de sa jeunesse, tout en baignant dans
2 Dans le sens où, comme nombre d'œuvres au tournant du XXI ${ }^{e}$ siècle, le livre se fonde sur la réutilisation d'une œuvre pré-existante, voire le détournement de celle-ci.

3 Pour ce travail, je me suis basée sur les éditions suivantes: Michael Cunningham, Les Heures, Paris, Belfond, 1999 [1998], trad. par Anne Damour; Virginia Woolf, Mrs Dalloway, Paris, Gallimard, 1994 [1925], trad. par MarieClaire Pasquier. Le titre du livre de Cunningham s'inspire directement de l'intitulé que Virginia Woolf souhaitait initialement donner à son roman. L'écrivain américain a utilisé de nombreuses sources de ce type, tant biographiques que littéraires, qui lui ont permis de constituer un solide réseau de citations et de références. Voir à ce propos la bibliographie de Richard J. Lane, Mrs Dalloway, Detroit/New York/San Francisco/Londres/Boston/Woodbridge, CT/ Gale Group, 2001, p. 225. Comme le montre Lane dans cet ouvrage, le roman de Woolf a suscité, bien avant le texte de Cunningham, maintes reprises, tant à l'opéra qu'au cinéma. 
4 "Pendant des années, le malaise resta enfoui, inavoué, dans l'esprit des femmes américaines. C'était une sensation étrange, un sentiment d'insatisfaction, une aspiration à autre chose que les femmes ressentirent au milieu du XXe siècle aux Etats-Unis. Toutes les femmes mariées résidant dans les grandes banlieues durent, seules, tenter de trouver un remède à ce malaise." (Betty Friedan, La femme mystifiée, Paris, Gonthier, 1964, p. 7 [1963]). Verbalisant ce qu'elle appelle le "problème qui n'a pas de mots " "The Problem that has no name" (titre du chapitre 1), à savoir le désenchantement des femmes au foyer après la Seconde Guerre mondiale, Friedan remet en question la version féminine du rêve américain en décrivant et décodant le vague à l'âme de femmes souvent plus aisées, instruites et diplômées que leurs consœurs des grands centres urbains, mais devenues des maîtresses de maison à plein temps au sein de villes-banlieues. L'impact international immédiat du livre, paru en 1963, a exercé une profonde influence sur la mobilisation des femmes et sur nombre de textes théoriques de la deuxième vague du féminisme américain (en provenance aussi bien de la branche libérale que radicale), tels Germaine Greer, The Female Eunuch, Londres, Paladin, 1971 [1970]; Eva Figes, Patriarchal Attitudes, Londres, Faber and Faber, 1970; Kate Millett, Sexual Politics, New York, Doubleday, 1970; Shulamith Firestone, The Dialectic of Sex, New York, Farrar, Straus and Giroux, 2003 [1970]. une ambiance de réminiscences. Le film se concentre pour sa part sur une même journée vécue par un trio féminin à trois époques différentes, réunies et marquées à divers niveaux par Mrs Dalloway: Woolf ellemême (Nicole Kidman), qui entame en 1923 l'écriture de son roman; Laura Brown (Julianne Moore), femme au foyer d'après-guerre, qui est une lectrice de ce livre; et l'éditrice Clarissa Vaughan (Meryl Streep), qui s'apparente si clairement à une incarnation contemporaine du personnage-titre de Woolf qu'elle est surnommée "Mrs Dalloway" par son entourage.

D’emblée, les passages dévolus à la journée de l'écrivaine la montrent s'attaquant dès le matin à la rédaction de Mrs Dalloway, dont elle rédige les premières lignes. Puis on la voit élaborer mentalement la suite de ce roman, lors d'une promenade ou, dans l'après-midi, pendant la visite de sa sœur accompagnée de ses trois enfants. Après une tentative avortée d'escapade à Londres, Woolf accepte, le canevas de son histoire enfin trouvé, de se coucher et de délaisser son manuscrit pour la nuit. Quant à Laura Brown, sa journée est scandée par la pénible confection, avec son fils en bas âge Richie (Jack Rovello), d'un gâteau d'anniversaire, par la lecture de Mrs Dalloway, et, surtout, par des événements qui renvoient tous à son mal de vivre. Ce personnage exemplifie en effet la naissance de la prise de conscience féministe américaine. Le "problème qui n'a pas de mots $\mathbf{4}$ dont souffre cette mère enceinte d'un deuxième enfant est en effet évoqué à travers plusieurs scènes, telles qu'une discussion avec une voisine (laquelle, après l'avoir écoutée résumer Mrs Dalloway, lui révèle sa profonde tristesse de ne pouvoir tomber enceinte et estime dès lors manquer ce qui aurait fait d'elle "une vraie femme»). Après s'être enfuie dans un hôtel pour y poursuivre la lecture du roman et essayer de s'y donner la mort, Laura revient sur sa décision et rentre à la maison célébrer comme convenu l'anniversaire de son époux avant de s'endormir, le vague à l'âme. Enfin, dans la troisième piste narrative

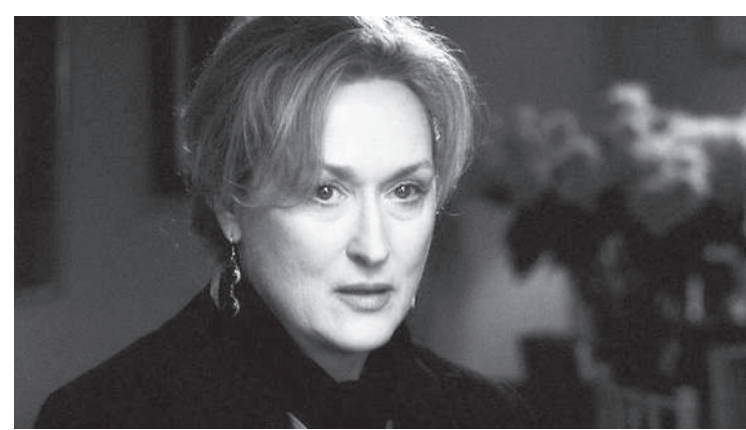

12

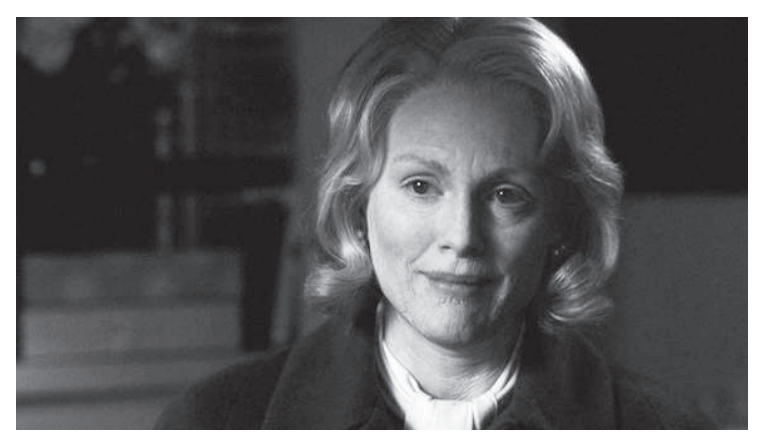

13 
du film, nous suivons Clarissa qui s'adonne aux préparatifs d'une fête qu'elle organise en l'honneur de son ami de longue date Richard (Ed Harris), un écrivain à succès. Elle fait une série de rencontres au cours desquelles certains protagonistes se réfèrent au dernier livre de Richard : la fleuriste, qui avoue ne rien avoir saisi au texte et émet quelques commentaires indiscrets au sujet du personnage féminin inspiré par Clarissa ; l'un des convives arrivé en avance, l'ex-compagnon de l'auteur, qui fustige la vacuité de l'ouvrage, en particulier sa dimension trop autobiographique pour une "fiction"; et en fin de journée, la mère du poète - figure assassinée dans le roman de Richard -, qui n'est autre que Laura Brown, la deuxième femme de l'histoire, qui réapparaît soudain après avoir abandonné son mari, son fils et sa fille un demi-siècle plus tôt. Ce dialogue permet de rendre plus explicite la démarche féministe de Laura, constamment associée à la lecture inspirante de l'œuvre de Virginia Woolf. La vieille dame formule les raisons de sa terrible décision de disparaître, d'échapper à cette mort lente pour refaire sa vie. Jusqu'alors reliés indirectement par des procédés comme le montage alterné ou l'usage de la musique, les principales protagonistes, ou du moins deux d'entre elles, se voient ainsi littéralement mises face à face, confrontées dans un même espace-temps (fig. 12-14). L'après-midi de Clarissa se termine par son passage chez Richard, malade et révolté, où elle assiste, médusée, à sa défenestration, geste fatal qui fonctionne à l'évidence comme un écho - masculinisé - du traumatisme suicidaire de Virginia sur lequel s'ouvrait le film.

Si Woolf évolue dans l'Angleterre de 1923, alors que Mrs Brown et Clarissa vivent aux Etats-Unis, respectivement à Los Angeles en 1949 et à New York en 1999, les trois univers et destins des femmes sont constamment mis en parallèle et entremêlés par l'alternance des séquences, voire à l'intérieur de celles-ci, des plans ${ }^{5}$. Immédiatement après le prologue, alors que défile le texte du générique, le début de la journée des

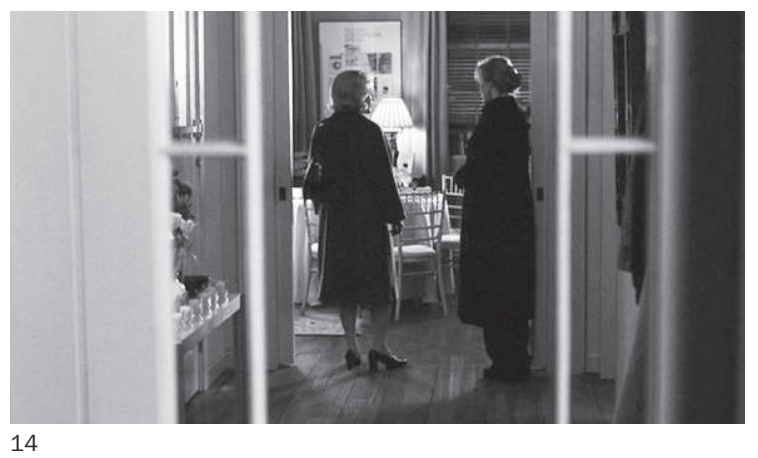

5 Le film de Stephen Daldry multiplie les échos, non seulement entre ces trois femmes, mais aussi entre une série de personnages secondaires inspirés par l'œuvre de Virginia Woolf. Parmi ceux-ci, je ne retiendrai que certains jeux de symétrie, de contraste ou d'inversion. 
6 Le bouquet est mis en série: les roses apportées par Dan, la gerbe fanée que déplace Clarissa et les violettes réarrangées par l'une des bonnes de Virginia se font écho. Le même principe est par exemple utilisé lors de la préparation de repas, notamment par des plans serrés sur des œufs que l'on casse chez Virginia, puis chez Clarissa.

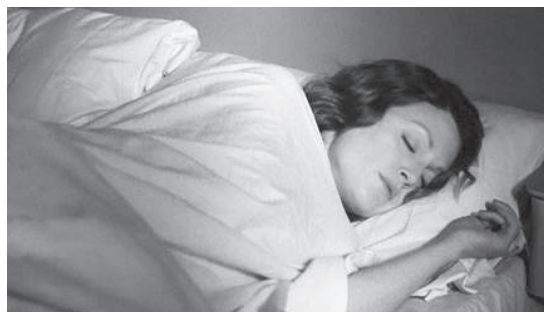

15

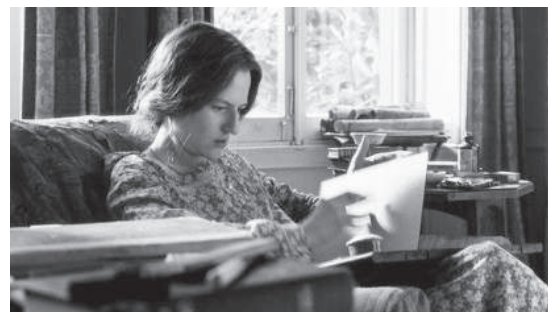

18

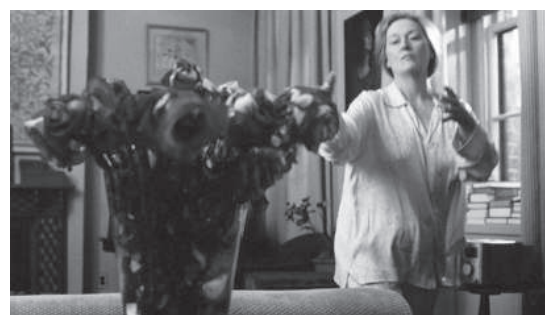

21

trois héroïnes prend ainsi la forme d'un montage élaboré, sans équivalent direct dans le livre. On débute par les actions respectives des trois conjoints, les protagonistes se réveillant progressivement dans chacune des séries (fig. 15-17) : Dan Brown pose des fleurs sur le bar, pendant que son épouse dort encore; Leonard Woolf traverse le jardin, pénètre dans la demeure, parle avec le médecin au sujet de Virginia; Sally marche dans les rues de New York, rentre à la maison. Se succèdent alors les gestes quotidiens effectués tour à tour par les personnages, mouvements qui les rapprochent en dépit des époques différentes: leur toilette, leur petit-déjeuner, puis, à des rythmes variés (la durée n'est pas la même pour toutes les femmes), les activités liées à la littérature (l'écriture, la lecture et l'édition, fig. 18-20). Un premier objet de transition, un bouquet de fleurs, scande cette séquence (fig. 21-23) 6 . Le bouquet permet ensuite d'associer Woolf prononçant les premières phrases de son roman

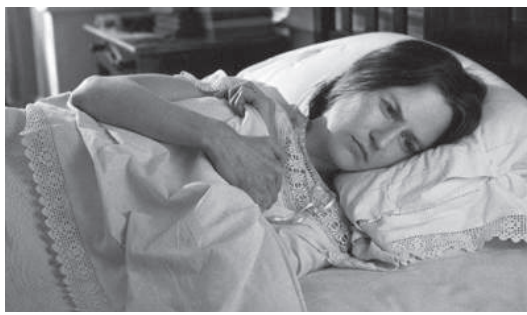

16

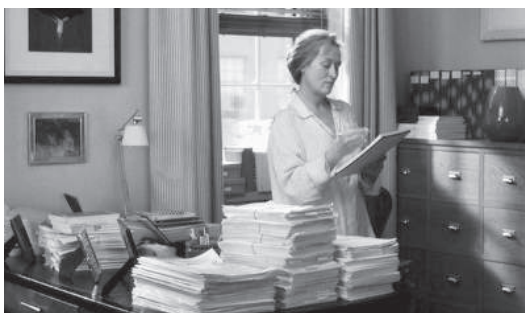

19

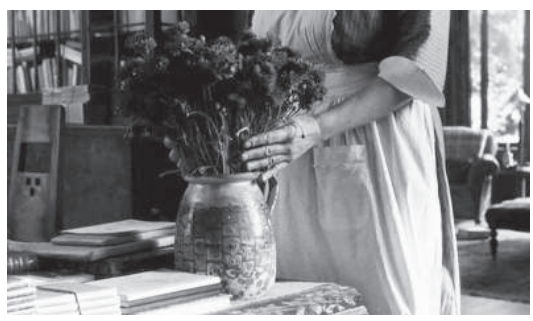

22

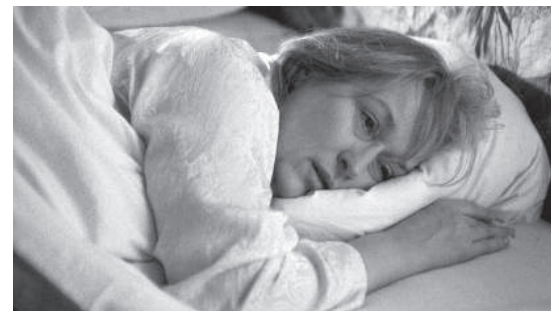

17

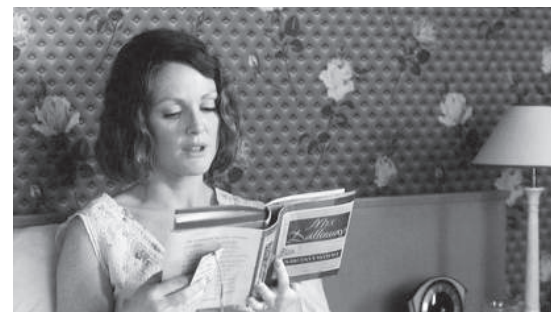

20

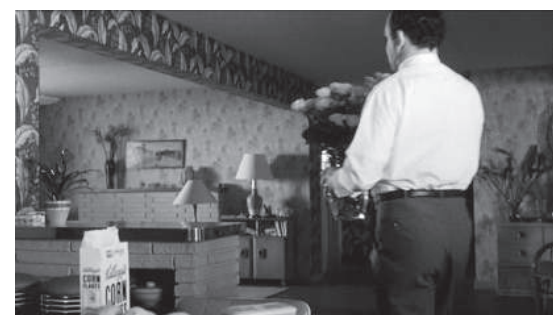

23 
à Mrs Brown qui ouvre justement le roman Mrs Dalloway pour en lire à voix haute l'incipit, puis à Clarissa Vaughan qui s'adresse à sa compagne ( «Sally, je crois que ce qui est des fleurs, je vais m’en charger» 7 ). Cette succession de plans très courts, sur le fond unifiant de la musique répétitive composée par Philip Glass, entrelace véritablement les parcours de ces trois femmes, annonçant les innombrables relations qui surgiront entre elles durant le film.

Si cette structure tout en croisements couvre l'essentiel du métrage, ce sont pourtant les images du prologue, situées dans une quatrième période succinctement évoquée, qui reviendront pour clore le film. Une fois les trois héroïnes couchées, deux plans reviennent en effet sur le suicide de 1941 pour montrer une dernière fois Woolf, de profil, au milieu de la rivière, avant qu'elle ne soit complètement immergée (fig. 8). En s'achevant sur la circularité d'un même motif, The Hours semble souligner le hiatus entre des journées "ordinaires» et le caractère exceptionnel des moments ultimes de l'existence. Mais ce constat n'est pas totalement exact, tant il semble difficile de considérer indépendamment ces deux "moments ", plus particulièrement vis-à-vis de la représentation de l'artiste, où la mise en exergue de cette mort joue un rôle central. L'œuvre tend d'ailleurs à le suggérer, peut-être involontairement, par une différence minime d'apparence physique entre la Virginia de 1941 et celle de 1923 que l'on voit ensuite à l'écran. Plus concrètement encore, les problèmes de santé préoccupants de Woolf sont évoqués très tôt, à peine quelques minutes après le prologue, lors d'une brève discussion entre Leonard et le médecin. Difficile donc de manquer cette mise en exergue des troubles de l'écrivaine qui traverse l'ensemble du film 8 .

\section{Le suicide de l'écrivaine et la mort du personnage}

La figuration de la mort de Woolf - moment auquel le film confère une importance cruciale -n'est pas dépourvue d'une certaine exaltation. En reprenant la structure romanesque établie par Michael Cunningham, Stephen Daldry et son scénariste David Hare cèdent apparemment à certaines connotations, empreintes de lyrisme romantique, qu'a pu susciter la persona de Virginia Woolf, dont la fin tragique a indéniablement renforcé l'accession à un statut mythique ${ }^{9}$. Si Cunningham indique, dans une note bibliographique située à la fin de son livre, que "Virginia Woolf, Leonard Woolf, Vanessa Bell, Nelly Boxall et d'autres encore apparaissent dans ce livre comme des personnages du roman", il souligne néanmoins sa volonté de "rendre aussi fidèlement que possible les détails de leur vie telle qu'elle s'est déroulée par une journée fictive de 1923 »10. Il n’évoque donc pas, à propos de la partie inspirée par la
7 Toutes les citations du film sont issues de la version française de The Hours (Paramount, dvd zone 1, 2002).

8 Plusieurs scènes évoquent la maladie de l'écrivain: pour Virginia, celles qui ont lieu avec son mari après la visite du médecin, et plus tard sur le quai de gare lors de sa tentative de fuite pour Londres, avec sa sœur Vanessa. Pour Richard, la première discussion avec Clarissa se concentre principalement sur son état de santé et les raisons qui motiveraient le poète à rester ou non en vie.

9 Sur l'écho médiatique suscité par la persona de l'écrivaine, voir Brenda R. Silver, Virginia Woolf Icon, Chicago/Londres, The University of Chicago Press, 1999 (surtout le chapitre "Take Seven. British Graffiti : Me, I'm afraid of Virginia Woolf and Sammy and Rosie get laid ", pp. 152173).

10 M. Cunningham, Les heures, op. cit., p. 225. 
11 Par exemple, The Routledge Companion to Feminism and Postfeminism, dont l'approche s'avère sensible aux questions de représentations, termine sa notule relative à Virginia Woolf en spécifiant les maux dont elle souffrait et la manière dont elle a disparu: "Les troubles psychologiques récurrents qui la hantaient depuis son enfance aboutirent à son suicide par noyade dans la rivière de l'Ouse en mars 1941" (Sarah Gamble (éd.), The Routledge Companion to Feminism and Postfeminism, Londres/New York, 1998, p. 340, ma traduction).

12 Ce film peut incontestablement être évalué à l'aune de certaines problématiques attachées à l'iconographie des créateurs, telle que déployée non seulement au cinéma, mais aussi dans la littérature et les essais historiques (comme les vies d'artistes). La plupart des biopics dédiés à des artistes s'ancre dans la figure romantique du génie individuel, travaillant de manière passionnée, instinctive, quelquefois même sans effort; ou encore dans une confrontation emblématique avec un artiste reconnu, souvent un mentor à dépasser. Cf. Rudolf et Margot Wittkower, Les enfants de Saturne, Paris, Macula, 1991 [1963]; Jürgen Felix (dir.), Genie und Leidenschaft. Künstlerleben im Film, St. Augustin, Gardez! Verlag, 2000; John A. Walker, Art and Artists on Screen, Manchester/ New York, Manchester University Press, 1993; Helmut Korte et Johannes Zahlten, Kunst und Künstler im Film, Hameln, C. W. Niemeyer, 1990.

13 "Et donc, prenant sa petite sœur pour confidente, il était parti pour Londres en laissant un mot idiot, comme en écrivent les grands hommes, et dont le monde prend connaissance par la suite, quand l'histoire de leurs luttes est devenue célèbre." (V. Woolf, Mrs Dalloway, op. cit., p. 172). biographie woolfienne, l'année 1941, bien que ce passage soit également présenté en tant que prologue du roman. Cette omission signale que le segment dévolu à la mort de l'écrivaine anglaise est à considérer à part au sein du roman The Hours, et, par extension, dans le film qui en est adapté. Même si Cunningham puise dans une source historique (la lettre d'adieu à Leonard, reprise intégralement dans le film), ce prologue relève principalement d'une création imaginaire tendant à projeter une image stéréotypée du personnage de Virginia Woolf. Il n'est certes de loin pas le premier à suivre cette voie: les circonstances de la mort de l'écrivaine sont fameuses et figurent en bonne place dans d'innombrables ouvrages monographiques ${ }^{\mathbf{1 1}}$. Non seulement cet épisode funeste entre directement en résonance avec l'œuvre de Woolf, qui développe en effet, à plusieurs reprises, la problématique du suicide comme celui d'une relation particulière à l'eau, mais, plus largement, il correspond à une image romantique de l'artiste encore très vivace de nos jours 12 .

Le suicide figure d'ailleurs dans Mrs Dalloway par le biais d'un personnage particulier, Septimus Warren Smith. Sa mort volontaire, qui clôt le texte de Woolf, est reprise et retravaillée dans le film The Hours. Redevable de la dynamique intertextuelle propre à Michael Cunningham, elle donne lieu à deux scènes: la noyade du prologue et la défenestration de l'écrivain Richard (sur laquelle se conclut d'ailleurs le roman). Si les velléités artistiques de ce protagoniste sont évoquées avec ironie par celle qui lui a donné vie - animé par la vocation de devenir poète, Septimus incarne pour Woolf la prétention de ceux qui pensent être géniaux $\mathbf{1 3}$-, son sort en tant que traumatisé de guerre fait par contre l'objet d'un grand respect. Seul personnage ayant réellement vécu et éprouvé l'expérience des combats meurtriers, Septimus demeure la victime des ravages psychologiques et physiques causés par la Grande Guerre, éprouvant plus spécifiquement d'affreuses crises de folie liées aux shell shocks. Ses affres, qui le poussent vers une mort atroce - en se jetant de la fenêtre, il s'empale sur des pointes métalliques et se croit alors assailli par les flammes - servent le propos d'une condamnation non seulement de la guerre, mais également de la médecine, que Woolf considère comme exclusivement porteuse de valeurs viriles issues du monde patriarcal. La maladie, et l'incompréhension que ne cesse de rencontrer Septimus, y compris de la part des médecins, sont reliées, dans The Hours, au personnage de Richard, atteint du sida et souffrant de crises aigues de dépression mélancolique. L'attribution de cet état d'âme troublé à un écrivain renvoie, une fois de plus, à la mythologie du créateur isolé en proie aux doutes les plus extrêmes et à l'introspec- 
tion morbide14. Même si la manière dont Richard se donne la mort fait sans doute référence à Septimus, ce personnage de The Hours redouble surtout, dans l'économie interne du récit, celui de Virginia, situé pour sa part dans les années 1920, puisque tous deux sont des artistes tourmentés qui décident de mettre fin à leurs jours. Peu avant de se laisser glisser le long de la fenêtre, Richard déclare d'ailleurs à Clarissa: "Je ne crois pas que deux êtres puissent être aussi heureux que nous l'avons été». Ces dernières paroles prononcées par le poète new-yorkais à son éditrice citent explicitement celles laissées par Virginia Woolf à son époux (et... éditeur) dans sa célèbre lettre d'adieu15. Pour autant, si la folie est associée dans Mrs Dalloway à un personnage masculin, elle devient, dans The Hours, un aspect central attribué à la personnalité de l'écrivaine elle-même. Ce déplacement mérite que je m’y arrête. En revenant sur les images sous-marines du prologue, sur ces plans où le corps de Virginia se mêle aux profondeurs obscures de la rivière, il me semble possible de déceler une autre facette de la représentation de Woolf en artiste maudite, par le biais d'une figure iconographique qui est a priori surprenante relativement à l'imaginaire social qui entoure l'écrivaine britannique: celle d'Ophélie.

\section{Virginia en Ophélie}

A première vue, en effet, rien ne permet d'associer la personnalité de Virginia Woolf à la malheureuse héroïne de Hamlet ${ }^{\mathbf{1 6}}$. Si le texte de Shakespeare, écrit au tournant du XVII ${ }^{e}$ siècle, décrit cette dernière comme une femme jeune, naïve et sans pouvoir, Virginia Woolf est, en 1941, âgée de 59 ans et jouit d'un fort prestige social. En "choisissant» cette mort-là, l'auteure du Voyage au phare a pourtant rendu possible son rapprochement avec le canon ophélien. Dans Le deuxième sexe, Simone de Beauvoir souligne notamment la prédilection des femmes pour le suicide par la noyade, puisqu'elles n'emploient «presque jamais les armes blanches ni les armes à feu [et] se noient beaucoup plus volontiers, telle Ophélie, manifestant l'affinité de la femme avec l'eau passive et pleine de nuit où il semble que la vie puisse passivement se dissoudre »17.

Archétype de la culture victorienne anglaise du XIX ${ }^{\mathrm{e}}$ siècle, Ophelia connut une immense fortune qui a inspiré en Occident tant la littérature, la peinture, la photographie $\mathbf{1 8}$ que la musique. Apparaissant dès la fin du XVIII e siècle dans l'art anglais, l'iconographie autour de cette figure connaît un essor prolifique qui se manifeste par d'innombrables représentations, du romantisme jusqu'à nos jours : toiles, lithographies déclinées dans la photographie de mode, en pochettes de disques, voire même en publicités. Richard Altick a par exemple montré la
14 Les deux scènes de dialogue entre Richard et Clarissa nuancent quelque peu le portrait de l'écrivain par le biais de ses propres commentaires empreints de cynisme: "J'ai eu ce prix [...] parce que je suis en train de craquer, mais que malgré tout je fais preuve de courage et qu'en plus, je m'accroche à la vie [...]; si j'étais en santé, tu crois que l'on me l'aurait donné? ".

15 De plus, lorsque Richard parle de son écriture, ses propos pourraient résonner avec l'art de Virginia Woolf: "Je voulais être écrivain, c'est tout [...]. Je voulais alors pouvoir décrire tout ce que contient un simple et court moment, comme décrire ces fleurs que tu portais dans tes bras, ou l'odeur de cette serviette, son apparence, sa texture, toutes nos émotions, les tiennes et les miennes, et l'histoire qui les englobe, qui nous étions alors, tout ce qu'il y a dans ce monde... [...] et j'ai échoué à cette tâche". En outre, dès leur rencontre de jeunesse, Richard surnomma Clarissa "Mrs Dalloway " en référence au personnage du roman de Virginia Woolf. II s'y réfère notamment dans la première scène les réunissant: "Oh! Mrs Dalloway, toujours à organiser des fêtes, parce qu'ainsi elle pense remplir le silence".

16 William Shakespeare, Hamlet, Paris, Flammarion, 1995, pp. 346-349 [François Maguin, éd. et trad.]. Dans cette pièce (1600-1601), le passage décrivant la mort d'Ophélie est situé aux vers 167 à 183 .

17 Simone de Beauvoir, Le deuxième sexe, vol. 2 [L'expérience vécue], Paris, Gallimard, 1949, p. 436.

18 D'ailleurs la grande tante de Virginia Woolf, la célèbre Julia Margaret Cameron, prit un cliché de Cyllene Wilson incarnée en Ophelia (Julia Margaret Cameron, Ophelia (Cyllene Wilson), 1867, photographie, sans dimensions). D'après Anne Higonnet, Cameron jouait auprès de Woolf le rôle à la fois d'«une excentrique et [d']un modèle à suivre", et la nièce fut la première à rééditer les travaux et à rédiger une préface pour la parution de l'ouvrage Victorian Photographs of Famous Men and Fair Women. Voir Anne Higonnet, "Femmes et images. Apparences, loisirs, subsistance", dans Geneviève Fraisse et Michelle Perrot (éd.), Histoire des femmes en Occident. IV. Le XIXème siècle, vol. 4, Paris, Plon, 2002 [1991], pp. 383-384. 
19 Richard Daniel Altick, "Hamlet", Paintings from Books. Art and Literature in Britain, 17601900, Colombus, Ohio State University Press, 1985, pp. 298-306; Katia Poletti, La mort d'Ophélie. Emergence, évolution et déclinaison d'un motif. Iconographie aux XVIII et XIXe siècles, mémoire de licence, Section d'Histoire de l'Art, Faculté des Lettres, Université de Lausanne, 2000 [non publié].

20 Lire Elaine Showalter, The Female Malady. Women, Madness and English Culture, 18301980 (1985), Londres, Virago Press, 1993, pp. 1-20 et E. Showalter, "Representing Ophelia: Women, Madness and the Responsabilities of Feminist Criticism ", dans Patricia Parker et Geoffrey Hartman (éd.), Shakespeare and the Question of Theory, New York/Londres, Methuen, 1985, pp. 77-94.

21 Dans son étude synthétique sur les principales "idoles de la perversité" au XIX siècle, Bram Dijkstra reproduit une multitude d'images de la faiblesse, de la fragilité et de la vulnérabilité féminine en insistant sur leur statut d'iconographie de la misogynie. Parmi les exemples qu'il cite, figurent des images d'Ophélie, où se révèle le plus clairement le caractère mortifère d'une tendance iconographique plus large. Bram Dijkstra, "The Cult of Invalidism; Ophelia and Folly; Dead Ladies and the Fetish of Sleep", Idols of Perversity. Fantasies of Feminine Evil in Fin-de-Siècle Culture, New York/Oxford, Oxford University Press, 1986, pp. 25-63.

\section{M. Cunningham, op. cit., pp. 15-16.}

23 John Everett Millais, Ophelia, 1852, huile sur toile, $76,2 \times 111,8 \mathrm{~cm}$, coins supérieurs arrondis, Londres, Tate Gallery.

24 M. Cunningham, op. cit., p. 15.

$25 \mathrm{Ibid}$. portée considérable de ce personnage shakespearien sur l'iconographie picturale ${ }^{\mathbf{1 9}}$, en considérant la mort tragique d'Ophélie comme l'un des motifs les plus populaires de la peinture anglaise durant le XIX ${ }^{\text {e }}$ siècle. D’autres protagonistes issus du théâtre de Shakespeare, comme Juliette, n'ont de loin pas suscité le même engouement, malgré un destin narratif similaire à celui de la bien-aimée de Hamlet. Il faut probablement en chercher les raisons du côté du contexte dans lequel se produit le suicide. Celui de Juliette se déroule sur une scène et non dans un décor naturel, mêlant eau et végétation, propice à des variations plastiques, des figurations au contenu symbolique beaucoup plus développé. Et si Beauvoir n'évoque pas, pour sa part, la perte de raison associée à la noyée, les représentations du tournant du siècle passé ont abondamment intégré ce paramètre spécifique. D’après Elaine Showalter, qui s'est justement appuyée sur l'analyse d'images illustrant la folie d'Ophélie, les productions visuelles ont d'ailleurs joué, dans la seconde moitié du $\mathrm{XIX}^{\mathrm{e}}$ siècle, un rôle majeur dans la construction théorique de la démence féminine 20 . Incarnant alors une sorte de prototype de la femme dérangée, Ophélie exprime à merveille l'idée reçue selon laquelle la folie devait être envisagée comme un mal spécifiquement féminin. Si cette représentation traduit les points de vue et les valeurs d'une époque, renvoyant plus à la construction victorienne de la féminité qu'à l'Ophélie de Shakespeare21, il apparaît néanmoins que le film de Stephen Daldry puise son inspiration dans cette tradition iconographique.

Certes, Michael Cunningham n'utilise jamais le nom d'“Ophélie» dans sa description de la noyée dans la rivière ${ }^{22}$, mais de nombreux éléments, inspirés tant par le texte shakespearien que par la riche iconographie qui en découle, permettent à mon sens d'affirmer que ce texte noue un dialogue, volontaire ou non, avec toute une série de références littéraires et visuelles. Si la place me manque dans ce travail pour déployer une analyse précise, The Hours rejoint l'esprit de quelques vers de Hamlet (dès 169), offrant lui aussi une image idéalisée d'un corps féminin abandonné et mourant. Les deux textes décrivent en effet la mort de leur héroïne en insistant sur la dimension poétique et peu morbide de la scène, sur l'harmonie entre le corps de la noyée et les éléments naturels qui l'entourent (eau et végétation). Si le texte de Cunningham fait penser à la bulle formée par la robe de la mourante de Millais 23 («le pan de son manteau de fourrure gonflant comme une vague derrière elle») ${ }^{\mathbf{2 4}}$, ou encore à la verdure et aux fleurs gisant sur le corps de la défunte du tableau de 1852 ("[1] es rubans d'herbe vert foncé s'accrochent à ses cheveux et à la fourrure de son manteau ") 25, la description de la noyée peut également être mise en relation avec deux autres Ophélie: celle d'Eugène 


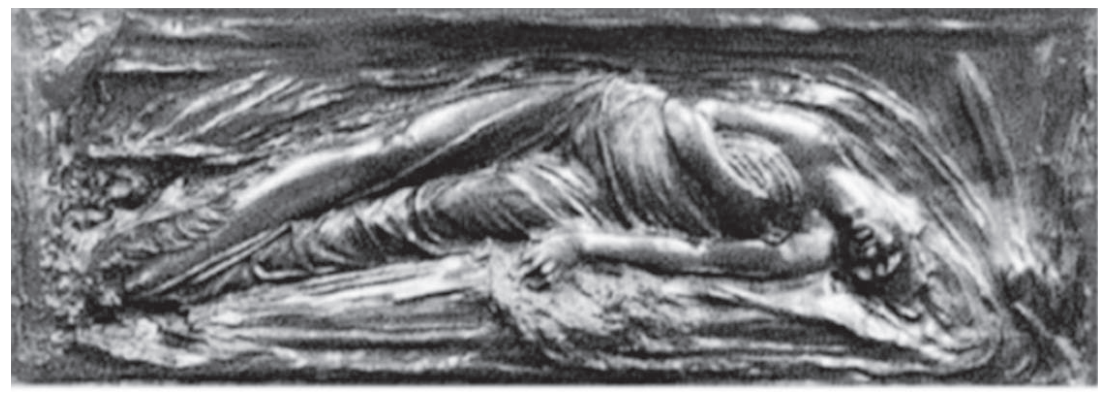

Delacroix (1838) 26, très proche de la référence à Hamlet, et celle, plus tardive, de Paul-Albert Steck (1895) 27. Ces images, mais surtout le bas-relief d'Auguste Préault datant de 1876 (fig. 24) illustrent bien l'émancipation du motif par rapport à Shakespeare, et peuvent aussi être rapprochées de la représentation filmique du suicide de Woolf dans The Hours. Outre sa focalisation sur la piste de la folie, l'adaptation cinématographique prolonge aussi la tradition visuelle d'Ophélie issue du XIX ${ }^{e}$ siècle, avec ces plans aquatiques qui donnent à voir la navigation légère de la noyée, totalement à l'horizontale, et qui entrent ainsi en résonance avec l'œuvre du sculpteur.

Dans le biopic, la voix over de Virginia vient se greffer sur les images du parcours de l'héroïne jusqu'à la rivière, ainsi que sur les derniers gestes de l'écrivaine, en alternance avec la découverte de la lettre d'adieu par son mari. La rédaction de cette missive est également visualisée et présentée sous la forme de quelques inserts, constituant une troisième série temporelle de ce prologue. Le montage cimente de la sorte l'association entre la lettre laissée par Virginia Woolf et son geste fatal, insistant dès lors sur l'hypothèse de la folie que l'énoncé de ce texte vient résolument étayer. Je le cite in extenso, comme le fait d'ailleurs le biopic.

«Mon chéri,

Je suis en train de sombrer dans la folie à nouveau, j'en suis sûre: je sais que nous n'arriverons pas au bout de ces horribles crises. Et cette fois je ne guérirai pas. Je recommence à entendre des voix, et n'arrive pas à concentrer mes pensées. Aussi vais-je faire ce qui me semble la meilleure chose à faire. Tu m'as rendue parfaitement heureuse. Tu as été pour moi ce que personne d'autre n'aurait pu être. Je ne crois pas que deux êtres eussent pu connaître si grand bonheur jusqu'à ce que commence cette affreuse maladie. Je ne peux plus lutter davantage, je sais que je gâche ta vie, que sans moi tu pourrais travailler. Et je sais que tu le feras. Tu vois, je n'arrive même pas à
24/ Auguste Préault, Ophélie, 1876, bas-relief, bronze, $72 \times 200 \times 20 \mathrm{~cm}$, Paris, Musée d'Orsay

26 Eugène Delacroix, La Mort d'Ophélie, 1838, huile sur toile, $38,1 \times 45,9 \mathrm{~cm}$, Munich, Neue Pinakothek.

27 Paul-Albert Steck, Ophélie, 1895, huile sur toile, $162 \times 98,5 \mathrm{~cm}$, Paris, Musée du PetitPalais. 
28 Cette traduction est issue de l'édition française du roman de Cunningham, op. cit., pp. 1314.

29 Si le film ne fait aucune allusion au contexte politique, le texte de Cunningham s'inscrit quant à lui plus précisément dans ce dernier. Tout en privilégiant la piste de la maladie, Cunningham lui-même évoque cette terreur peu avant le suicide ("On est en 1941. Une autre guerre vient d'éclater. [...] des bombardiers grondent dans le ciel, bien qu'elle cherche en vain à apercevoir des avions") ou encore la peur de l'échec en écriture: "Elle, elle a échoué. Elle n'est pas du tout un écrivain, en réalité; elle n'est qu'une excentrique douée" (M. Cunningham, op. cit., p. 11). Par ailleurs, Marie-Paule Vigne énumère les différentes tentatives de suicide effectuées par l'écrivaine avant cette noyade (une première fois, Virginia Woolf se serait jetée d'une fenêtre, une deuxième fois, elle aurait absorbé du véronal). De même, quelques jours avant la noyade, son mari l'aurait rencontrée, "trempée et hagarde", celle-ci prétendant avoir "glissé dans un chenal ". II semble donc effectivement que la mort de Woolf ait résulté d'une décision mûrement réfléchie, et non pas d'un accès de pure folie. Voir Leonard Woolf, The Journey Not the Arrival Matters, Hogarth, 1969, cité par Marie-Paule Vigne, Le thème de l'eau dans l'œuvre de Virginia Woolf, Bordeaux, Presses Universitaires de Bordeaux, 1984, pp. 508-509. écrire correctement. Je n'arrive pas à lire. Ce que je veux dire, c'est que je te dois tout le bonheur de ma vie. Tu t'es montré d'une entière patience avec moi et indiciblement bon. Tout le monde le sait. Si quelqu'un avait pu me sauver, c'eût été toi. Tout m'a quitté excepté la certitude de ta bonté. Je ne veux pas continuer à gâcher plus longtemps ta vie. Je ne crois pas que deux personnes auraient pu être plus heureuses que nous l'avons été.

$$
\mathrm{V} . » 28
$$

Cette lettre témoigne certes de l'immense respect et de l'amour porté par Virginia Woolf à son époux, mais elle souligne aussi les troubles qui guettent l'écrivaine. D'emblée, elle parle elle-même de "folie", d'"affreuse maladie», décrit ses maux, "les voix» entendues, le fait de ne plus pouvoir dompter son esprit, de ne plus parvenir «à concentrer (s)es pensées", à écrire ou à lire. Sans espoir, elle évoque à plusieurs reprises l'impression de ne plus voir d'issue aux "horribles crises", juge impossible la guérison et a le sentiment d'être totalement abandonnée. La détermination qui transparaît dans ces derniers mots exprime un choix inexorable face à la maladie, mais se réfère aussi à d'autres inquiétudes profondes, comme le tour terrible pris par la guerre - l'Angleterre vit alors dans la terreur d'une invasion nazie - ou encore les difficultés et les échecs inhérents à l'exercice de la littérature. Sur les motifs l'ayant poussée au suicide, outre la peur de la maladie (comme en témoigne cette lettre, Virginia Woolf était effectivement sujette à des crises nerveuses très fréquentes, et était angoissée à l'idée de perdre la raison), il ne faut pas négliger le contexte de la Seconde Guerre mondiale, qui a suscité de fortes craintes chez l'écrivaine, dont le mari, Leonard, était juif 29.

D'une certaine façon, c'est justement pour insister sur la valeur «hors norme» de la personnalité artistique de Virginia Woolf que le suicide de l'écrivaine est ainsi mis en exergue dans The Hours. Le roman et le film débutent en effet là où s'est interrompue la trajectoire de Woolf, comme pour y trouver les traces d'un dernier acte créateur, à l'image de la mise en scène par l'artiste de sa propre mort. Cette perspective n'est pas nouvelle: de nombreuses études se sont effectivement penchées sur la biographie et l'œuvre de Virginia Woolf pour y déceler les signes avant-coureurs de sa noyade, en particulier à travers l'analyse de son obsession pour l'eau et la place occupée dans ses ouvrages par la question du suicide. Pour ma part, il est difficile de ne pas être tentée, devant une nouvelle au titre aussi énigmatique que La fascination de l'étang, d'y percevoir l'annonce de la manière dont l'artiste choisira de disparaître: 
«Mais si l'on s'asseyait au milieu des joncs pour regarder l'étang - les étangs exercent une curieuse fascination, on ne sait laquelle - [...]. Bien des gens avaient dû y venir au fil de leur vie, au fil des âges, laisser une pensée dans l'eau, lui poser une question, comme on le faisait soi-même en ce soir d'été. Peut-être était-ce le secret de sa fascination: il retenait dans ses eaux toutes sortes de rêves, de plaintes, de confidences, non pas imprimés ou dits à voix haute mais à l'état liquide, flottant les uns sur les autres, presque désincarnés. [...] Le charme de l'étang venait de ce que, les gens partis, leurs pensées étaient restées et, sans leurs corps, entraient vagabonder le temps qui leur plaisait, libres, liantes et amicales dans l'étang commun.»30

Cet attachement pour l'eau s'articule donc chez Woolf à un espace où s'estompent les contours rigides du monde matériel et où les pensées, désincarnées, s'épanouissent sans aucune restriction. De l'élément liquide se dégage le plus souvent la nostalgie d'un monde idéal, comme immergé et éloigné du temps, une source primordiale d'affection, de vie et de bonheur, que l'écrivaine associe plus particulièrement aux lieux où elle vécut ou passa ses vacances $\mathbf{3 1}$.

\section{"Dieu! Qu'elle est difficile à traduire ! "32}

Malgré cette possible interférence avec l'univers propre à Virginia Woolf, la place nodale attribuée à l'évocation du suicide dans The Hours - le film est comme clôturé par l'événement, qui devient le point de départ et d'arrivée du récit - encourage inévitablement une lecture biographique, qui s'avère incompatible avec la temporalité particulière préconisée par Woolf dans ses propres romans. En effet, The Hours s'oppose aux conceptions de l'écrivaine elle-même en matière de création littéraire. En mettant en exergue le suicide, on lui attribue une valeur primordiale : c'est à la lumière de ce moment-clé que le reste du film sera vu et envisagé. Une telle construction romanesque entre en contradiction avec celle adoptée par Woolf dans Mrs Dalloway, un roman où la narratrice évite de privilégier un facteur de compréhension au détriment d'un autre, de baliser les possibles significations. La dimension téléologique de The Hours répond à un principe opposé, le film s'ouvrant et se refermant sur le suicide de son personnage central.

En débutant par la constitution d'une sorte d'icône mortuaire, le biopic donne le sentiment que l'existence de sa protagoniste peut être appréhendée comme un tout, une tension permanente animée par la folie créatrice. Dans l'œuvre même qui se voit là réinterprétée, Mrs Dalloway, Virginia Woolf s'est certes aussi fixée pour objectif de construire une protagoniste capable d'accéder à une véritable représentation
$30 \mathrm{~V}$. Woolf, La fascination de l'étang, Paris, Seuil, 1990 [traduit par Josée Kamoun], pp. 157-158.

31 J. R. Noble, op. cit., p. 110, cité par MariePaule Vigne, op. cit., p. 507.

32 Roger Fry, le 21 décembre 1926, à propos des épreuves de Time Passes, dans Virginia Woolf, Le temps passe, Ed. Le bruit du temps [traduit par Charles Mauron], 2010 [1926], postface, p. 83. 
33 Voir par exemple la préface de Marguerite Yourcenar pour l'édition française du roman Les vagues, qu'elle a traduit de l'anglais en 1937. Virginia Woolf, Les vagues, Paris, Le Livre de Poche, 1993 [1931], pp. 5-12.

34 Virginia Woolf, Une chambre à soi, Paris, 10/18, 1992 [A Room of One's Own, 1929], pp. $65-67$. d'elle-même. Mais cette exigence découle de la volonté explicite de créer des personnages féminins échappant aux mythes à l'aune desquels on les représente et renvoyant le plus possible à des existences "ordinaires", marquées par une plus grande complexité de caractère. Rompant avec la génération des Edwardians, l'écrivaine s'accorde là à un courant de pensée des premières années du $\mathrm{XX}^{\mathrm{e}}$ siècle qui procède à la remise en cause de l'unité de l'individu et de la linéarité du temps (à des titres divers, y participent tant Henri Bergson que James Joyce ou Sigmund Freud). Virginia Woolf transpose ces réflexions dans le cadre de ses préoccupations littéraires grâce à la technique du stream of consciousness. Par l'utilisation de ce "flux de conscience »33, le personnage se voit envisagé sous ses différentes facettes. Dans Mrs Dalloway, l'absence d'une narratrice omnisciente au profit d'une variété infinie de points de vue qui se révèlent quelquefois paradoxaux permet autant de dégager un éventail d'opinions extérieures à la protagoniste que de pénétrer son intériorité en accédant à ses sentiments et sensations. Inversant le préjugé récurrent à l'endroit des femmes mondaines, perçues comme superficielles, ce personnage apparait, sous la plume de Woolf, comme une bourgeoise complexe, intelligente et tout en nuances, à la profondeur sans cesse renouvelée. Ce souci d'échapper aux clichés associés au sexe féminin se situe au cœur des réflexions développées dans l'essai Une chambre à soi, publié en 1929. L'écrivaine y décrit avec ironie la manière biaisée dont les écrivains ont représenté les femmes jusqu'à son époque:

"C'est certes un monstre étrange, celui que l'on conçoit en lisant tout d'abord les historiens, puis les poètes, un vermisseau qui aurait des ailes d'aigle, l'âme de la vie et de la beauté en train de hacher menu quelque morceau de lard dans sa cuisine. Mais ces monstres, si amusants soient-ils pour l'imagination, n'ont pas d'existence réelle.»34

Si la personnalité de Virginia Woolf peut encore, de nos jours, paraître hors du commun en raison de son statut d'écrivaine et de son union sans enfants qui la propulsaient en marge de l'idéal prôné par la bourgeoisie du XIX ${ }^{e}$ siècle, toute insistance sur sa mort contribue à rapporter cette singularité au stéréotype de l'«artiste maudit», qui caractérise en particulier, pour la période contemporaine, les images sociales de célèbres femmes créatrices. A mon sens, l'amalgame entre Ophélie et Virginia participe de la perpétuation d'un mythe, d'un effacement de déterminations singulières au profit de conventions iconographiques. Malgré l'attention documentée et la sensibilité que manifestent Cunningham et Daldry à l'égard de l'œuvre de Virginia Woolf, l'assimilation implicite de l'écrivaine à Ophélie conduit à envisager cette der- 
nière d'une manière complètement opposée à la démarche préconisée par la romancière anglaise.

La mise en exergue de la persona de Virginia Woolf, déjà effectuée par Cunningham, constitue donc une trahison de l'esprit moderniste féministe affiché par la créatrice, pour qui le sens d'un personnage devrait être produit par l'instant. En développant visuellement ce motif, les plans aquatiques de The Hours pourraient pointer pour leur part une autre perception de l'écrivaine. En effet, la représentation sous-marine de la noyée rejoint aussi, dans une certaine mesure, la pensée de Gaston Bachelard, qui théorise le "complexe d'Ophélie» comme archétype de "l'imagination créatrice»35. Cette idée a marqué l'exégèse woolfienne. Aussi le critique Keyes relie-t-il la manière dont est décédée celle qui rédigea Les vagues à sa méthode de travail littéraire, en comparant le fonctionnement de sa pensée à une rivière ( Her river-mind»36). Pour sa part, Viviane Forrester a signalé l'«obsession» de l'eau chez Virginia Woolf, cette eau «qui la fascine et qui [en fin de compte] se referme sur son corps» $\mathbf{3 7}$. Ces citations, recueillies parmi tant d'autres, sont données par Marie-Paule Vigne dans son étude spécifiquement dévolue à la place occupée par ce thème chez l'auteure britannique. Elle démontre la portée considérable du discours consistant à articuler la fin tragique de Woolf avec le rôle endossé par l'eau dans ses écrits, comme si sa mort venait confirmer ou donner plus de sens à son travail littéraire. Ce rapport est explicitement établi par Jean Guiguet:

«Qu'elle ait choisi de se noyer de préférence à toute autre mort, n'est peut-être qu'un témoignage illusoire: il est cependant difficile de n'y pas voir un geste qui rassemble et soudain donne son sens à cet univers dans son univers: il enlève à ces symboles, ces images, ces métaphores, ces motifs, ce qui peut s'y être glissé de littérature pour les charger en destinée.» $\mathbf{3 8}$

Les notices biographiques concernant Woolf évoquent donc très souvent le suicide par noyade et encouragent ainsi à établir le lien que choisira de développer notamment Daldry dans sa représentation du corps sans vie emporté par le courant. Cette perception n'est d'ailleurs pas prête de prendre fin, comme en témoigne la réédition récente de 25 nouvelles réunies sous l'intitulé La fascination de l'étang. Si ce titre est bien celui d'un des textes recueillis, le fait de sélectionner ce titre-là pour l'ensemble du recueil renvoie explicitement à la problématique mortifère qui m'occupe ici. Le résumé figurant sur la quatrième de couverture du livre indique explicitement que "ces nouvelles sont un peu l'éprouvette où se dépose l'œuvre de Virginia Woolf, comme loin des contraintes, depuis des années de jeunesse jusqu'au dernier
35 Gaston Bachelard, L'eau et les rêves, Paris, José Corti, 1942, p. 118.

36 Marie-Paule Vigne, Le thème de l'eau dans l'œuvre de Virginia Woolf, Lille, Université de Lille III Atelier national de reproduction des thèses, 1984, p. 4.

37 Viviane Forrester, Virginia Woolf, Paris, Ed. De la Quinzaine littéraire, 1973, p. 112, p. 7.

38 Jean Guiguet, Virginia Woolf et son œuvre, Paris, Didier, 1962, p. 401. 
$39 \mathrm{~V}$. Woolf, La fascination de l'étang, op. cit.

40 De nombreux plans de la main écrivant la lettre viennent scander la séquence (voir fig. 18). mois où l'appel des eaux dormantes est venu couvrir toutes les autres voix »39.

\section{Voix de Woolf et flux de conscience}

L'idée énoncée dans cette citation s'actualise parfaitement dans la version filmique de The Hours. En effet, dès sa noyade, la voix over de l'écrivaine s'interrompt. Au sein du prologue, cette association entre l'acte d'écriture et l'énonciation verbale est clairement posée à travers la série visuelle alternant le départ de Virginia vers l'Ouse, l'écriture préalable des mots d'adieu à Leonard et l'arrivée subséquente de ce dernier à la maison. La voix over surgit précisément sur le premier plan serré de la main de Woolf plongeant sa plume dans l'encrier: "Mon chéri, je suis certaine...»40. Sans que jamais, dans cette partie, on ne visualise la source de cette lecture, la voix over entame l'énoncé in extenso de la lettre destinée au mari. Sitôt le dernier mot prononcé, le silence s’impose - non seulement par l'arrêt de la parole, mais aussi par la cessation de la musique minimaliste de Philip Glass, qui avait démarré dès l'arrivée de la protagoniste au bord de la rivière - et, enfin, on perçoit l'immersion ultime du visage de l'écrivaine. Avec ce suicide se produit donc la disparition simultanée d'une créatrice et de sa puissante imagination.

Recourant encore à ce principe à la fin du film, la voix over de Virginia s'adresse une dernière fois au mari, prolongeant de la sorte la lettre d'adieu du prologue. Les mots "Mon chéri, regarder la vie en face, toujours, regarder la vie en face et la reconnaître pour ce qu'elle est. Et enfin, la reconnaître et l'aimer pour ce qu'elle est. Et puis, y renoncer» sont prononcés sur des images détaillant chacune des trois protagonistes au moment du coucher: Mrs Brown, vieille dame émue du geste de tendresse que lui a accordé la fille de Clarissa (incarnant la nouvelle génération libérée du passé); Woolf alitée (plan en plongée sur son visage sérieux et pensif); enfin, Clarissa finalement sereine, que l'on voit s'approcher d'une porte vitrée, la fermer, et, avant de pénétrer dans sa chambre, éteindre une à une les lampes du couloir, l'obscurité envahissant alors l'écran et permettant un raccord avec l'épilogue. Sur un plan large décrivant Woolf de profil, entrant dans la rivière et pénétrant progressivement dans l'eau, la voix over réapparaît une dernière fois pour proférer les derniers mots du film: «Léonard, toujours, toutes ces années ensemble, pour toujours ces années. Pour toujours, l'amour. Et toujours les heures", et stoppe précisément au moment du fondu au noir.

A l'image du stream of consciousness préconisé par Woolf, le dispositif vocal insiste sur la force créatrice de son écriture. Le surgissement de son 
texte ou de ses pensées est, presque toujours, traduit à voix haute par le personnage (que cette émission sonore soit off ou synchronisée avec sa source). Si la lecture des mots destinés à Leonard est donnée à entendre par le biais de la voix over de Woolf, le processus d'écriture du roman Mrs Dalloway, qui s'étire durant tout le film, bénéficie lui aussi de ce traitement. Les phrases prononcées par l'écrivaine servent par conséquent d'éléments structurants non seulement pour la séquence d'ouverture et de clôture, mais également pour l'ensemble du biopic. Avec le recours aux raccords dans le montage alterné et l'utilisation enveloppante de la musique, l'usage de la voix (celle de Woolf, parfois aussi celle des deux autres protagonistes) constitue une autre stratégie filmique pour rendre plus fluide une navigation rapide entre les existences des trois femmes, et pour souligner le processus de création de l'écrivaine, c'est-à-dire l'élaboration progressive du roman.

Dès la fin du générique, soit après la séquence du réveil, l'écrivaine apparaît d'emblée préoccupée. La journée est elle-même clôturée par le travail mental de Woolf sur son prochain livre autour du surgissement, au petit matin, de la première idée ("Léonard, je crois que j’ai ma première phrase») $\mathbf{4 1}$ à l'achèvement, le soir, du canevas de Mrs Dalloway $\mathbf{4 1}$ Ma traduction.
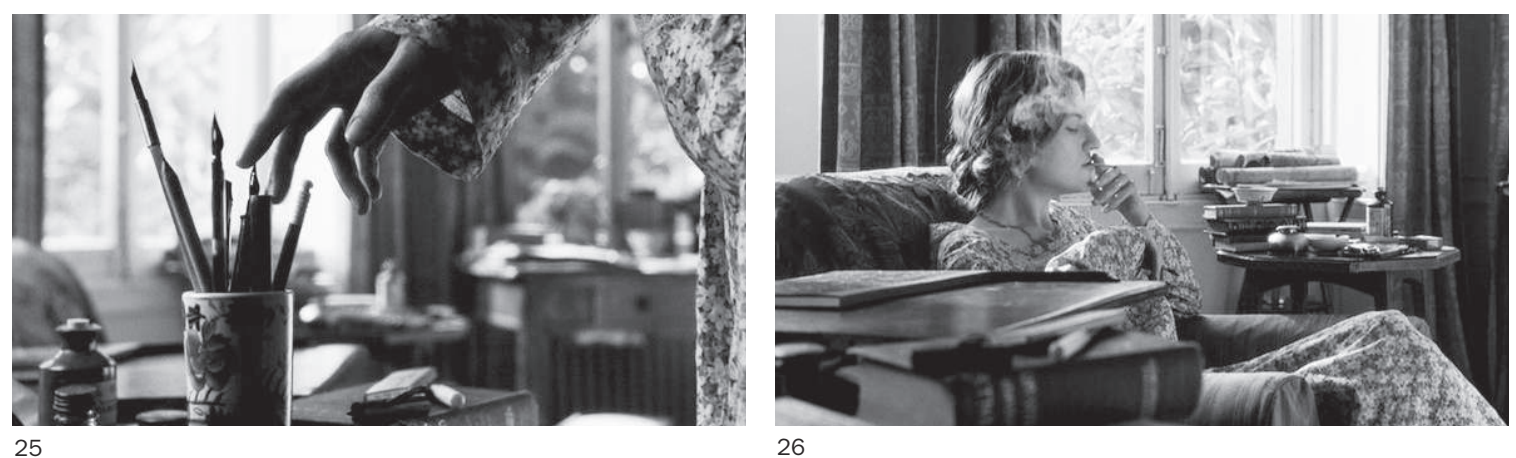
26
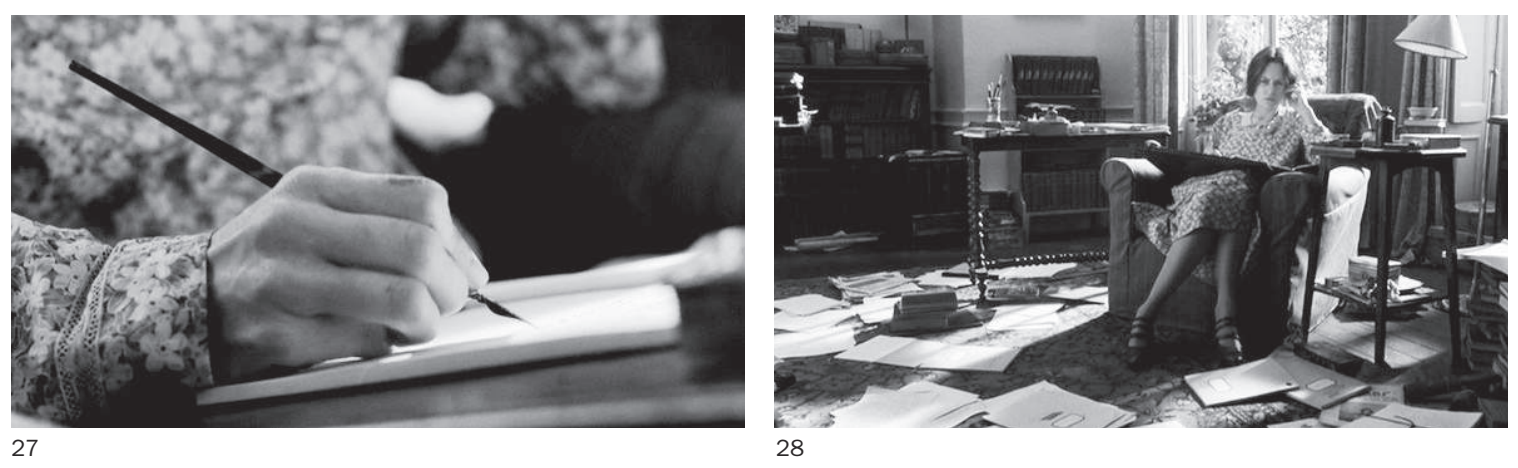


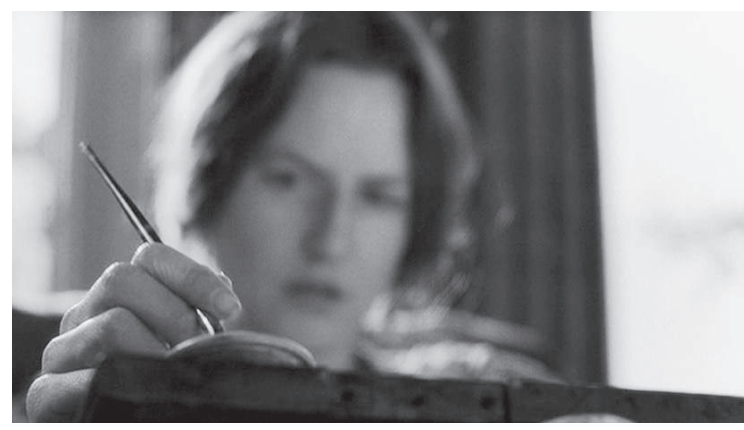

29

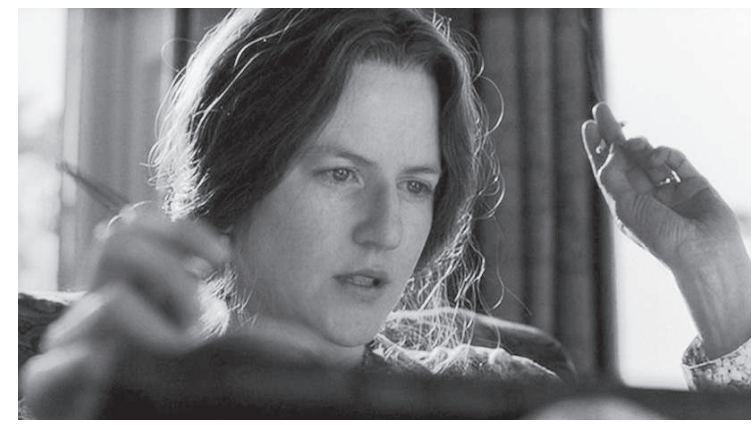

30
42 Dans Journal d'un écrivain (Paris, 10/18, [trad. par Germaine Beaumont] 1977 [1953]), édition établie par Léonard Woolf à partir des 26 volumes du Journal de son épouse dont il a extrait "tout ce qui, pratiquement, relève de son travail d'écrivain ", Virginia Woolf mentionne à de nombreuses reprises ses doutes à propos de l'élaboration du livre "qu' [elle], appelle Les Heures" (mardi 19 juin 1923, p. 104): "Je draine laborieusement mon cerveau à l'intention de Mrs. Dalloway. Mais je ne ramène que des sceaux à moitié plein et je n'aime pas cette sensation. J'écris trop vite (16 août 1922, p. 90)". Elle évoque son achèvement dans sa note du samedi 13 décembre 1924 (p. 124): "Je vais maintenant au triple galop et je re-tape entièrement Mrs. Dalloway depuis le début [...]. Une bonne méthode je crois, c'est comme si on passait sur l'ensemble un pinceau humide pour unifier les parties qui avaient séché séparément. Réellement, sincèrement, je trouve que c'est le plus satisfaisant de mes romans (mais je ne l'ai pas encore relu de sang-froid). Les critiques diront que c'est décousu, parce que les scènes de la folie ne se raccordent pas directement aux scènes Dalloway [...]. Mais, comme il me semble l'avoir déjà dit, j'ai l'impression qu'il m'a fait descendre dans les strates les plus profondes et les plus riches de mon cerveau. Je peux maintenant écrire, écrire, et encore écrire. C'est la sensation la plus délicieuse qui soit au monde".
("Je vais aller me coucher [...]. Tout le reste est clair. Les grandes lignes de l’histoire sont tracées. Il ne reste qu'une chose. Je dois décider de la destinée de Mrs Dalloway»). Habitée par l'écriture de son personnage, Woolf est constamment montrée en train d'écrire ou de réfléchir à haute voix. Cette énonciation verbale révèle les diverses étapes de l'élaboration sinueuse du roman, à l'instar des déplacements de Virginia d'un endroit à un autre, qui peuvent être interprétés comme les reflets de son cheminement créatif intérieur 42. C'est d'abord installée dans son sofa et choisissant sa plume que Woolf, cigarette aux lèvres, prononce la première phrase: "Mrs Dalloway dit qu'elle se chargerait elle-même d'acheter les fleurs». Faisant suite au montage fragmenté qui insiste sur la mise au travail de Woolf (fig. 25-28) - séquence qui s'achève par une vue en plongée montrant l'accumulation, sur le sol, de manuscrits et de pages noircies -, un plan se stabilise et se fixe alors sur le visage de l'écrivaine (dont la main tient la plume en amorce, fig. 29-30) pour accentuer la scansion verbale de la phrase initiale du roman ; celle-ci est immédiatement prolongée en un double écho temporel: on assiste à la lecture orale

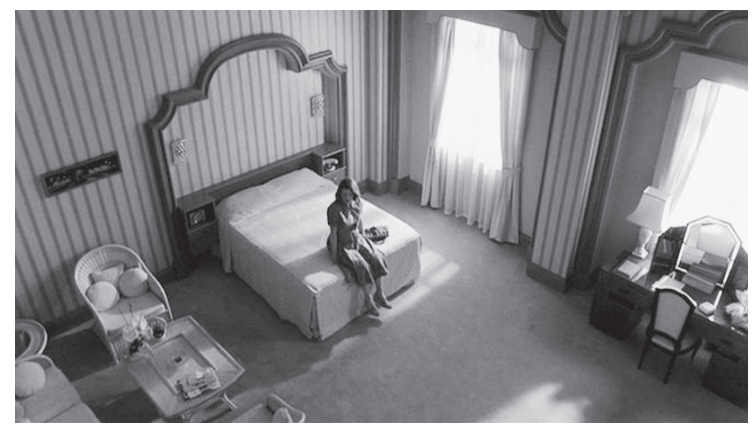

31 
de ces mêmes premières lignes par Mrs Brown, ainsi qu'à l'allusion qui en est faite, aussitôt après, dans une injonction in de Clarissa.

Quelques minutes plus tard, le film revient sur Woolf en train de poursuivre l'élaboration de son livre (à nouveau, plans serrés sur sa main écrivant, son visage absorbé). Mais, cette fois, le moment où elle verbalise une nouvelle idée textuelle («La vie toute entière d'une femme dans une seule journée, une seule journée») fait l'objet d'un traitement visuel particulier, sous la forme d'un rapide zoom avant sur elle. Ce mouvement de caméra est repris dans le plan suivant, qui montre Mrs Brown dans sa cuisine, toujours accompagnée par la parole ininterrompue de Virginia: «Et durant cette journée, toute sa vie». Plus loin, après la longue scène opposant Clarissa et l'écrivain Richard, le film revient sur le pendant féminin de celui-ci dans l'économie du film, Virginia. Encore une fois, elle est représentée en pleine concentration sur son travail de création (resserrements du cadre sur la bouche qui aspire une bouffée de cigarette, sur la main qui écrit, sur le visage, sur le manuscrit, sur l'encrier...), disant distinctement: "C’est ce jour-là, précisément ce jour-là que son destin lui apparut clairement». Dérangée par la bonne - qui est exaspérée de l'absence trop manifeste de la maîtresse de maison -, Woolf quitte alors sa chambre. A partir de cet instant, on la suit, poursuivant dans divers lieux l'invention de Mrs Dalloway. Ainsi, lors d'une courte ballade à Richmond, assise sur un banc, elle énonce l'idée narrative cruciale à laquelle elle vient d'aboutir : «Elle mourra. Oui, elle va mourir. C'est ça qui va arriver. C'est ça. Elle va se tuer. Elle va se tuer pour quelque chose qui semble sans importance». Au plan visuel, un lien direct est alors effectué avec Mrs Brown, qui tente pour sa part de s'encourager à préparer un gâteau d'anniversaire.

Ce rapprochement audiovisuel entre les pensées de Woolf et la vie de la mère de famille d'après-guerre est surtout établi dans une longue

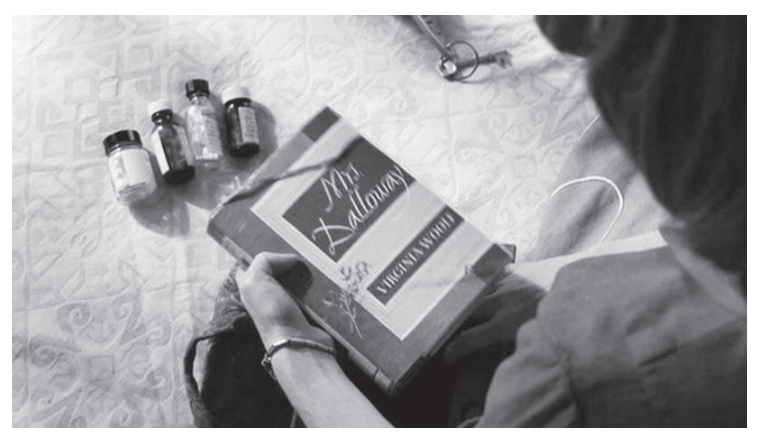

32

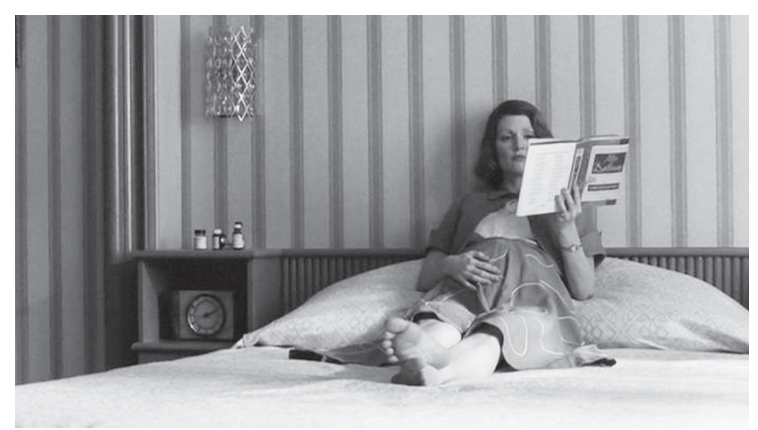

33 

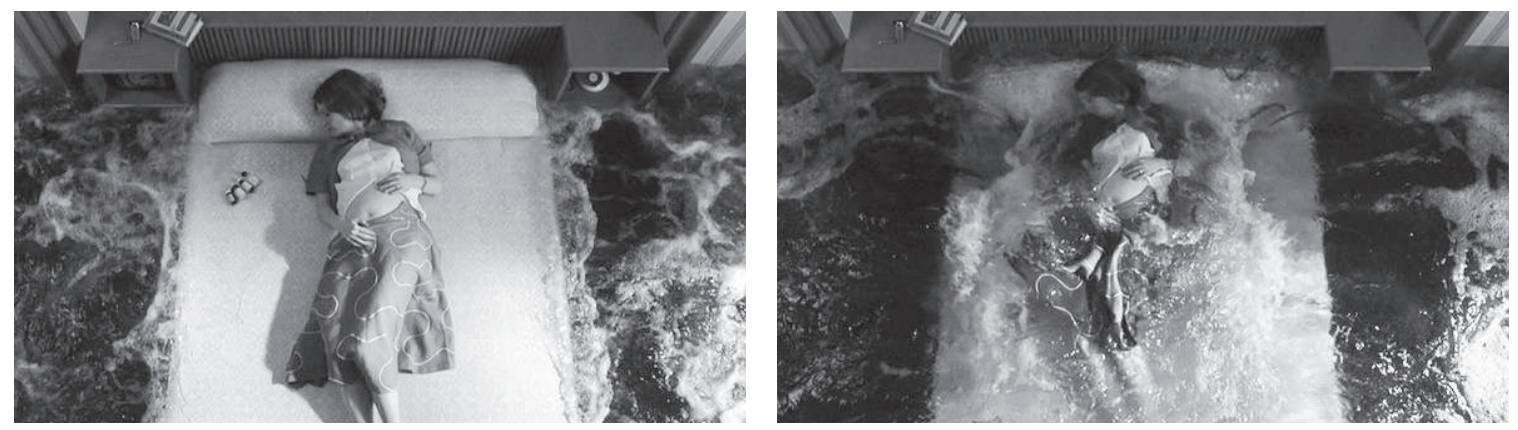

34

35
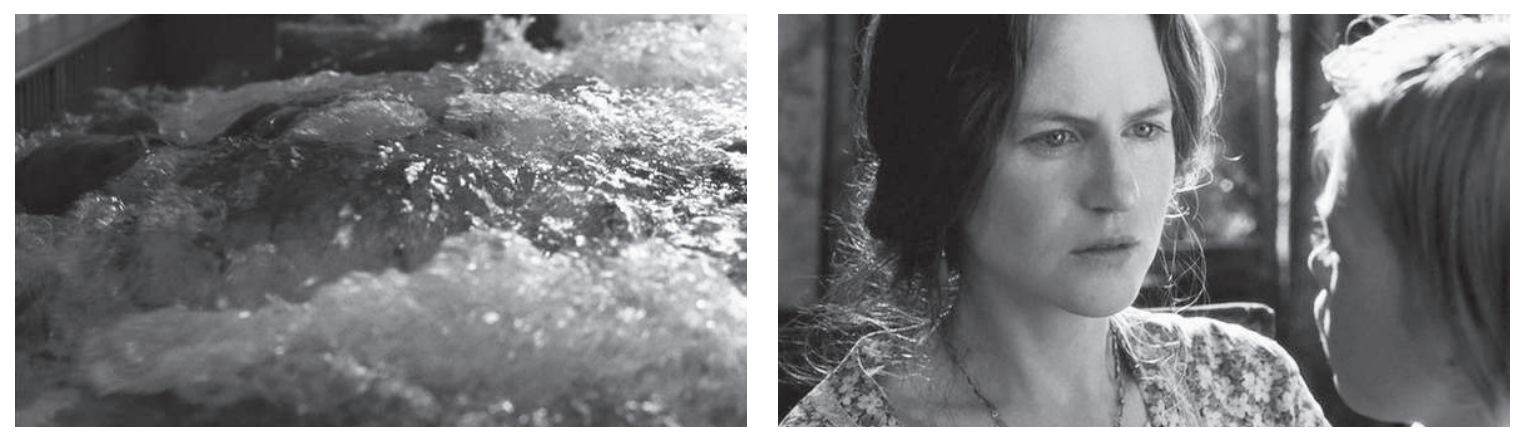

37

séquence de montage alterné entre, d'un côté, l'écrivaine qui échafaude mentalement, pendant la visite de sa sœur et ses neveux, une réflexion autour de la mort de son héroïne et, de l'autre, Mrs Brown, installée dans une chambre d'hôtel, qui se couche sur le lit, près de ses barbituriques et de son exemplaire de Mrs Dalloway. L'enchevêtrement entre la méditation créative de Woolf et son actualisation dans l'histoire de Mrs Brown est structuré en trois parties.

Tout d'abord, lorsque Laura, alitée, reprend sa lecture, la voix over de Woolf s'y superpose. La réflexion de l'écrivaine à propos de son personnage et du sens de l'existence se poursuit dans une alternance de plans entre les deux protagonistes (fig. 31-33). Ensuite, dès qu'une vue montre Laura refermant le livre et le déposant à ses côtés, la suspension de la lecture est soulignée par le silence de Woolf. Un raccord intervient sur la sœur de Virginia interpellant celle-ci, perdue dans ses pensées (auxquelles nous n'avons, nous aussi, plus accès): "Es-tu toujours avec nous ?". Ne réussissant à extraire Woolf de sa rêverie, Vanessa s'adresse alors à sa fille: "Ta tante a vraiment beaucoup de chance Angelica... car elle possède deux vies, elle a sa propre vie et elle a en plus celle de son livre. Elle est vraiment choyée». Les songes de l'auteure semblent alors 
incarnés par une plongée qui expose Mrs Brown, allongée sur le lit, progressivement submergée par une inondation qui rappelle évidemment la noyade de l'écrivaine. Mais avant que l'eau n'ait totalement englouti le personnage, un plan serré montre Virginia répondant enfin à sa nièce : "J'étais sur le point de tuer mon héroïne, mais j’ai décidé de ne pas le faire» (fig. 34-37).

Enfin, ce volte-face se traduit par le réveil de Laura, que l'on voit revenir brutalement à la réalité (il n'y a plus d'eau autour d'elle), reprenant sa respiration comme si elle venait de suffoquer, s'asseyant sur le bord du lit, se touchant le ventre (allusion à sa prochaine responsabilité maternelle) et renonçant à mourir : "Non, c'est trop dur» (en voix in). Se focalisant alors sur Woolf, la caméra capture le rire gêné des neveux 43 s'esclaffant face à l'attitude absente de leur tante, qui conclut: "Angelica, je crains qu'à la place je sois contrainte de tuer quelqu'un d'autre».

En soirée, Leonard, assis au coin du feu, interpelle son épouse avant qu'elle ne remonte travailler dans son bureau, l'interrogeant sur l'identité du personnage destiné à mourir, ainsi que sur les motivations de ce choix. Virginia explique alors que certaines morts permettent au reste de l'humanité de pouvoir jouir plus profondément de la vie et que, dans
43 Les propos de Quentin Bell, neveu de l'écrivaine, divergent de cette vision doucement ironique. Par exemple: "Elle faisait les délices des enfants. L'annonce «Virginia vient pour le thé, était comme une brise chaude et capricieuse soufflant du sud-ouest et apportant avec elle une sorte de joie confondante". Quentin Bell, Virginia Woolf Biographie II 1912-1941, Paris, Stock, [trad. par Francis Ledoux], 1974 [1972], p. 155.
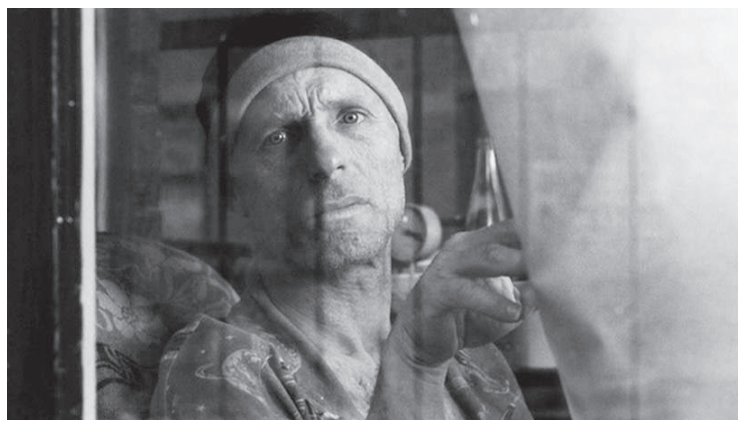

38

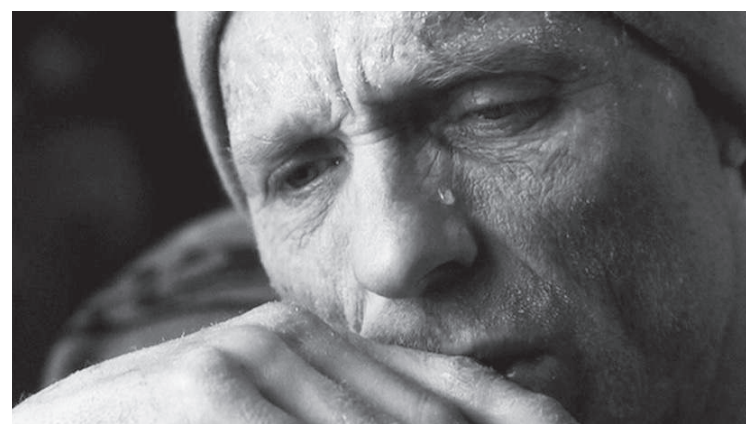

40

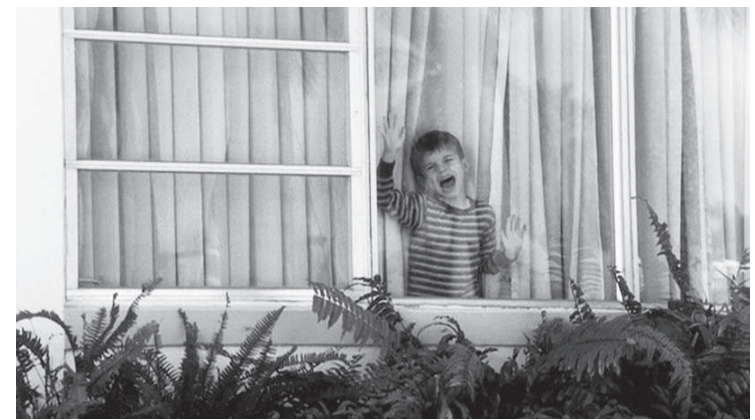

39

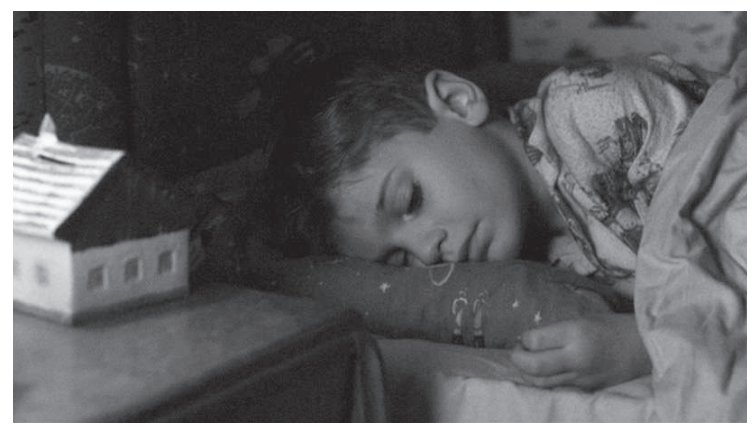

41 
44 "Leonard: Pourquoi faut-il que quelqu'un meure? Dans ton livre, tu dis qu'il faut que quelqu'un meure. Pourquoi? Est-ce une question stupide? [...]. Virginia: Pas du tout. Léonard: Alors? Virginia: II faut que quelqu'un meure afin que les autres apprécient la vie encore plus. Par contraste. Léonard: Et qui mourra? Dis-le moi. Virginia: Le poète mourra. Le visionnaire.".
$45 \mathrm{~V}$. Woolf, Une chambre à soi, op. cit. son roman, c'est le poète, le visionnaire, qui est appelé à disparaître $\mathbf{4 4}$. Explicitant par le montage l'une des thématiques centrales du film, c'est-à-dire la généalogie d'"artistes maudits" (à la trajectoire fatale de Septimus dans Mrs Dalloway répondent, dans The Hours, celles de Woolf elle-même et de Richard), le biopic établit alors, au moment où Virginia évoque son personnage "condamné», un lien visuel immédiat avec l'écrivain Richard, montré à l'époque où il est encore le petit garçon de Laura (on ne connaît pas formellement, à ce moment-là, son identité future): dans sa chambre à coucher, à la fin de la journée, l'enfant ne s'endort pas et pleure en silence (fig. 38-41).

Si l'assemblage de ces derniers épisodes, loin du bureau, parvient à représenter le processus créatif de Woolf sans que l'artiste ne soit montrée écrivant une seule ligne, la fin du film présente d'une manière synthétique le fruit de cette élaboration. A la nuit tombante, Woolf a réintégré sa chambre. Assise face à sa machine à écrire, juste avant qu'elle n'aille se coucher, l'écrivaine annonce alors à son mari avoir trouvé le canevas de Mrs Dalloway (fig. 42-43). Au final, l'artiste est montrée à nouveau dans cet espace important, en fonction d'une iconographie située à mi-chemin entre l'atelier (lieu de travail) et la pièce privée (lieu de l'intimité). Cette ambivalence découle directement d'une dernière image de l'artiste que je veux convoquer ici, et qui s'ancre cette fois pleinement dans les réflexions de Virgina Woolf sur les conditions spécifiques de la création féminine.

\section{Une chambre à soi}

Les divers lieux physiques que Woolf occupe ou traverse se rattachent en effet à l'idée de cheminement artistique, plus particulièrement vis-àvis de l'écriture d'un roman. Dans son célèbre essai Une chambre à soi 45, Virginia Woolf a réfléchi aux conditions favorables qui permettent aux femmes de son époque d'écrire en toute indépendance. Pour pouvoir

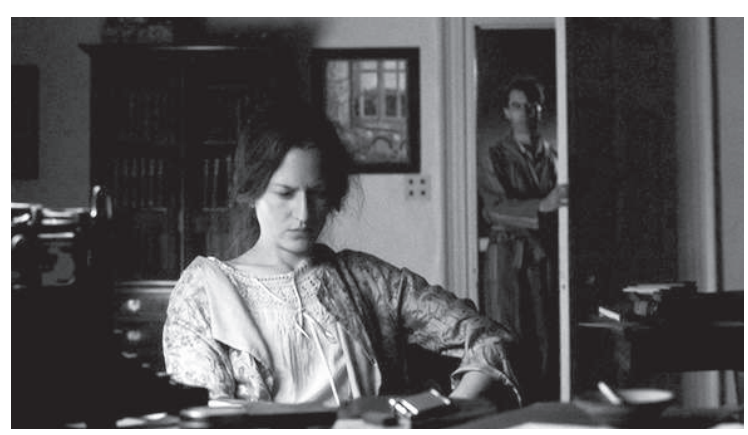

42

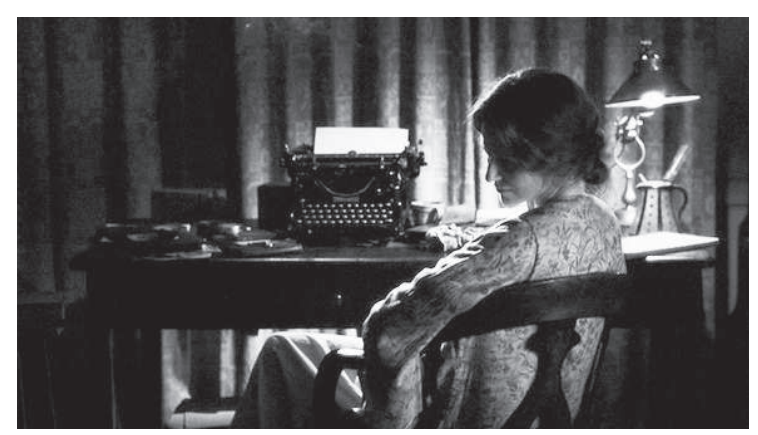

43 
créer, une artiste doit, selon l'auteure anglaise, bénéficier non seulement d'une certaine indépendance financière, mais aussi d'un espace personnel qui garantit sa liberté.

Ces propos de Virginia Woolf sont certes incarnés dans le film par la présence de l'héroïne à son bureau, mais aussi par le parcours de Mrs Brown qui, pour échapper à son mal-être et parvenir à son émancipation, se met en quête de sa "chambre à soi». Ainsi cette ménagère se se réfugie-t-elle d'abord dans la solitude d'une chambre d'hôtel, où elle demande explicitement à ne pas être dérangée, avant d'abandonner au final son foyer pour s'installer dans un pays où elle trouvera un travail. La littérature joue un rôle incitatif essentiel dans ce parcours, puisque, outre la lecture de Mrs Dalloway, c'est un emploi de bibliothécaire qui va, comme elle l'affirme explicitement, la "sauver» de sa morne existence.

Par la présentation de cette trajectoire d'émancipation, le film interprète donc le célèbre essai en fonction d'une conception toute matérielle. Au-delà de cette première lecture, il déploie néanmoins une appréhension parallèle, plus abstraite, d'Une chambre à soi. En extrayant Woolf d'un strict lieu de création, et en suivant les divers déplacements de l'écrivaine, le film offre une sorte de matérialisation de l'espace mental nécessaire à l'acte créateur. En cela, The Hours répond aux mêmes préoccupations qui innervent d'autres biopics contemporains dévolus aux femmes artistes. La question de l'atelier y est en effet essentielle en ce qui concerne les peintres ou les sculptrices, comme dans Frida [Kahlo] (Julie Taymor, 2002), Artemisia [Gentileschi] (Agnès Merlet, 1997) ou encore Camille Claudel (Bruno Nuytten, 1998). Si Leonard, occupé dans l'imprimerie (la célèbre Hogarth Press) à éditer des ouvrages, estime que son épouse a de la chance de pouvoir ainsi, en matinée, aller se balader, il rappelle aussi une dichotomie à l'œuvre dans les films représentant des artistes féminines. En fustigeant fréquemment la dérive institutionnelle de l'art (les ateliers aux allures de manufacture, les pressions insupportables des commanditaires publics ou privés, l'accaparement administratif, la compromission avec les intérêts d'autrui...) comme source d'un tarissement de l'inspiration "géniale», les films considérés tendent souvent à justifier indirectement l'isolement de leurs héroïnes dans des ateliers personnels, souvent extérieurs à leur domicile principal : Gentileschi se crée sa "tente» à elle devant la maison familiale, Kahlo circule dans des espaces divers en fonction de son état physique ou psychologique et Claudel finit par ne plus faire la différence entre son habitat et son atelier. Ces films cherchent à opérer une sorte de fusion entre l'artiste et son œuvre à travers l'effacement de l'atelier traditionnel $\mathbf{4 6}$. The Hours pousse cette tendance à la disparition jusqu'à dissoudre sa

46 Voir Rachel Noël, Créatrice ou Créature: Représentations de femmes artistes à l'écran (Artemisia Gentileschi, Camille Claudel, Frida Kahlo), Mémoire DEA en Etudes Genre, sous la direction de la Prof. Claire Lise Schwok, Universités de Genève et de Lausanne, Facultés des SSP, 2008. 
47 Voir Virginia Woolf, Les fruits étranges et brillants de l'art, Paris, Editions Des Femmes, 1983 [traduit par Sylvie Durasranti]; ainsi que V. Woolf, L'écrivain et la vie et autres essais, Paris, Editions Payot \& Rivages, 2008 [traduit par Elise Argaud].

$48 \mathrm{~V}$. Woolf, "Les femmes et la fiction", dans Les fruits étranges et brillants de l'art, op. cit., pp. 9-19.

49 ld., pp. 53-201.

50 /d., p. 31. protagoniste dans la matière liquide. Cependant, loin de dépeindre une créatrice irrémédiablement tombée dans la folie, l'épilogue présente une femme désormais apaisée qui ne cite plus sa lettre d'adieu, comme dans le prologue, mais un extrait évoquant le passage du temps, naviguant ainsi de la dimension personnelle à celle, plus éternelle, de la création artistique accomplie.

Si The Hours n'évoque guère les réflexions de Virginia Woolf relatives à son statut de romancière et d'intellectuelle ${ }^{47}$, ni ne fait allusion à ses nombreuses critiques littéraires touchant notamment aux rapports entre "Les femmes et la fiction $\mathbf{4 8}$ ou à ses études consacrées à des auteures telles que Eliza Haywood, Mary Wollstonecraft, Jane Austen, Charlotte Brontë, Elisabeth Barrett Browning, Elisabeth Gaskell, George Eliot, Dorothy Richardson ou Marie de Roumanie 49, le film parvient toutefois à échapper, ne serait-ce que partiellement, au douloureux constat énoncé par Woolf dans son essai Des professions pour les femmes édité en 1931, où elle estimait que "durant encore fort longtemps, sans doute, aucune femme ne pourra tenter d'écrire sans trouver devant elle un spectre» $\mathbf{5 0}$. 